\title{
The Bipolar Choquet Integral Representation
}

\author{
Salvatore Greco, Fabio Rindone ${ }^{\dagger}$ \\ Department of Economics and Business, University of Catania, \\ Corso Italia, 55, 95129 Catania, Italy
}

\begin{abstract}
Cumulative Prospect Theory of Tversky and Kahneman (1992) is the modern version of Prospect Theory (Kahneman and Tversky (1979)) and is nowadays considered a valid alternative to the classical Expected Utility Theory. Cumulative Prospect theory implies Gain-Loss Separability, i.e. the separate evaluation of losses and gains within a mixed gamble. Recently, some authors have questioned this assumption of the theory, proposing new paradoxes where the Gain-Loss Separability is violated. We present a generalization of Cumulative Prospect Theory which does not imply Gain-Loss Separability and is able to explain the cited paradoxes. On the other hand, the new model, which we call the bipolar Cumulative Prospect Theory, genuinely generalizes the original Prospect Theory of Kahneman and Tversky (1979), preserving the main features of the theory. We present also a characterization of the bipolar Choquet Integral with respect to a bi-capacity in a discrete setting.
\end{abstract}

Key words: Cumulative Prospect Theory, Gains-Loss Separability, biWeighting Function, Bipolar Choquet Integral.

JEL Classification D81 · C60

*salgreco@unict.it, Tel. +39 095.7537733 - Fax +39 095-7537510
${ }^{\dagger}$ frindoneo@unict.it, Tel. +39 095.7537733 - Fax +39095 7537510 


\section{Introduction}

Cumulative Prospect Theory (CPT) of Tversky and Kahneman (1992) is the modern version of Prospect Theory (PT) (Kahneman and Tversky (1979)) and is nowadays considered a valid alternative to the classical Expected Utility Theory (EUT) of Von Neumann and Morgenstern (1944). CPT has generalized EUT, preserving the descriptive power of the original PT and capturing the fundamental idea of Rank Dependent Utility (RDU) of Quiggin (1982) and of Choquet Expected Utility (CEU) of Schmeidler $(1986,1989)$ and Gilboa (1987). In recent years CPT has obtained increasing space in applications in several fields: in business, finance, law, medicine, and political science (e.g.,Benartzi and Thaler (1995); Barberis et al. (2001); Camerer (2000); Jolls et al. (1998); McNeil et al. (1982); Quattrone and Tversky (1988)). Despite the increasing interest in CPT - in the theory and in the practice - some critiques have been recently proposed: Levy and Levy (2002); Blavatskyy (2005); Birnbaum (2005); Baltussen et al. (2006); Birnbaum and Bahra (2007); Wu and Markle (2008); Schade et al. (2010). In our opinion, the most relevant of these critique concerns the GainLoss Separability (GLS), i.e. the separate evaluation of losses and gains. More precisely, let $P=\left(x_{1}, p_{1} ; \ldots ; x_{n}, p_{n}\right)$ be a prospect giving the outcome $x_{i} \in \mathfrak{R}$ with probability $p_{i}, i=1, \ldots, n$ and let $P^{+}\left(P^{-}\right)$be the prospect obtained from $P$ by substituting all the losses (gains) with zero. GLS means that the evaluation of $P$ is obtained as sum of the value of $P^{+}$and $P^{-}: V(P)=V\left(P^{+}\right)+V\left(P^{-}\right)$. Wu and Markle (2008) refer to the following experiment: 81 participants gave their preferences as it is shown below (read $\mathcal{H}>\mathcal{L}$ "the prospect $\mathcal{H}$ is preferred to the prospect $\mathcal{L}^{\prime \prime}$ )

$$
\begin{gathered}
\mathcal{H}=\left(\begin{array}{c}
0.50 \text { chance } \\
\text { at } \$ 4,200 \\
0.50 \text { chance } \\
\text { at } \$-3,000
\end{array}\right)>\left(\begin{array}{c}
0.75 \text { chance } \\
\text { at } \$ 3,000 \\
0.25 \text { chance } \\
\text { at } \$-4,500
\end{array}\right)=\mathcal{L} \\
{[52 \%]} \\
\mathcal{H}^{+}=\left(\begin{array}{c}
0.50 \text { chance } \\
\text { at } \$ 4,200 \\
0.50 \text { chance } \\
\text { at } \$ 0 \\
{[15 \%]}
\end{array}\right)<\left(\begin{array}{c}
0.75 \text { chance } \\
\text { at } \$ 3,000 \\
0.25 \text { chance } \\
\text { at } \$ 0 \\
{[85 \%]}
\end{array}\right)=\mathcal{L}^{+}
\end{gathered}
$$




$$
\mathcal{H}^{-}=\left(\begin{array}{c}
0.50 \text { chance } \\
\text { at } \$ 0 \\
0.50 \text { chance } \\
\text { at } \$-3,000
\end{array}\right)<\left(\begin{array}{c}
0.75 \text { chance } \\
\text { at } \$ 0 \\
\\
0.25 \text { chance } \\
\text { at } \$-4,500
\end{array}\right)=\mathcal{L}^{-}
$$

As can be seen, the majority of participants preferred $\mathcal{H}$ to $\mathcal{L}$, but, when the two prospects were split in their respective positive and negative parts, a relevant majority prefers $\mathcal{L}^{+}$to $\mathcal{H}^{+}$and $\mathcal{L}^{-}$to $\mathcal{H}^{-}$. Thus, GLS is violated and $\mathrm{CPT}$ cannot explain such a pattern of choice. In the sequel we will refer to this experiment as the "Wu-Markle paradox".

In the CPT model the GLS implies the separation of the domain of the gains from that of the losses, with respect to a subjective reference point. This separation, technically, depends on a characteristic $S$-shaped utility function, steeper for losses than for gains, and on two different weighting functions, which distort, in different way, probabilities relative to gains and losses. We aim to generalize $\mathrm{CPT}$, maintaining the S-shaped utility function, but replacing the two weighting functions with a bi-weighting function. This is a function with two arguments, the first corresponding to the probability of a gain and the second corresponding to the probability of a loss of the same magnitude. We call this model the bipolar Cumulative Prospect Theory (bCPT). The bCPT will allow gains and losses within a mixed prospect to be evaluated conjointly. In the next we discuss our motivations. The basic one, stems from the data in Wu and Markle (2008) and Birnbaum and Bahra (2007). Both of these papers, following a rigorous statistical procedure, reported systematic violations of GLS. Moreover, if we look through the Wu-Markle paradox showed above, we understand that the involved probabilities are very clear, since they are the three quartiles $25 \%$, $50 \%$ and $75 \%$. Similarly, the involved outcomes have the "right" size: neither so small to give rise to indifference nor so great to generate unrealism. Now suppose to look at the experiment in the other sense, from non mixed prospects to mixed ones. The two preferences $\mathcal{L}^{+}>\mathcal{H}^{+}$and $\mathcal{L}^{-}>\mathcal{H}^{-}$, under the hypothesis of GLS, should suggest that $\mathcal{L}$ should be strongly preferred to $\mathcal{H}$. Surprisingly enough, $\mathcal{H}>\mathcal{L}$. What happened? Clearly, the two preferences $\mathcal{L}^{+}>\mathcal{H}^{+}$and $\mathcal{L}^{-}>\mathcal{H}^{-}$did not interact positively and, on the contrary, the trade-off between $\mathcal{H}^{+}, \mathcal{H}^{-}$and $\mathcal{L}^{+}, \mathcal{L}^{-}$was in favor of $\mathcal{H}$. These data, systematically replicated, seem to suggest that a sort of Gain Loss Hedging (GLH) appears in the passage from prospects involving only gains or losses to mixed ones. When the GLH 
phenomenon is intense enough to reverse the preferences, i.e. $\left(\mathcal{L}^{+}>\mathcal{H}^{+}\right.$and $\left.\mathcal{L}^{-}>\mathcal{H}^{-}\right)$and also $\mathcal{H}>\mathcal{L}$, then GLS is violated. Thus, the first motivation of the paper is to show how bCPT is able to capture, at least partially, these erroneous predictions of CPT. A second motivation for proposing bCPT, stems from the consideration that, in evaluating mixed prospects, it seems very natural to applicate a trade-off between possible gains and losses. This, corresponds to assume that people are more willing to accept the risk of a loss having the hope of a win and, on the converse, are more careful with respect to a possible gain having the risk of a loss. Psychologically, the evaluation of a possible loss could be mitigated if this risk comes together with a possible gain. For example, the evaluation of the loss of $\$ 3,000$ with a probability 0.5 in the prospect $H=(0,0.5 ; \$-3,000,0.5)$ could be different from the evaluation of the same loss within the prospect $L=(\$ 4,200,0.5 ; \$-3,000,0.5)$, where the presence of the possible gain of $\$ 4,200$ could have a mitigation role. Why should be the overall evaluation of a prospects only be the sum of its positive and negative part? The last motivation has historical roots and involves the revolution given to the development of PT. Since when the theory has been developed (Kahneman and Tversky (1979)), a basic problem has been to distinguish gains from losses. However, in the evolution of decisions under risk and uncertainty, the majority of data, (e.g. Allais (1953); Ellsberg (1961); Kahneman and Tversky (1979)) regarded non-mixed prospects. Many authors (e.g. Luce (1999, 2000); Birnbaum and Bahra (2007); Wu and Markle (2008)) pointed that the mixed case is still a little understood domain.

This paper is organized as follow. In section 2 we describe the bCPT, starting from the CPT. In section 3 we present several bi-weighting functions, generalizing well know weighting functions. Section 4 is devoted to the relationship between CPT and bCPT. In section 5 we extend bCPT to uncertainty. Our main result, the characterization of the bipolar Choquet integral, is developed in section 6. We discuss some "coherence condition" in section 7 and we concludes in section 8 . The appendixes contain all the proofs and tests of bCPT on the previous data reported in the literature about the GLS violation. 


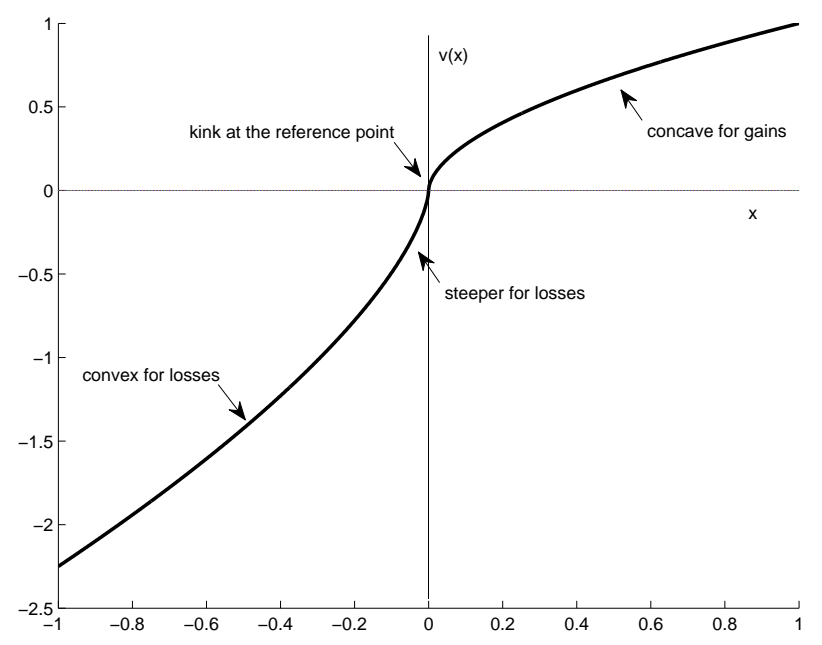

Figure 1: CPT utility function

\section{From CPT to bCPT}

\subsection{Two different approaches}

The most important idea in CPT is the concept of gain-loss asymmetry: people perceive possible outcomes as either gains or losses with respect to a reference point, rather than as absolute wealth levels. The characteristic S-shaped utility function $^{1}$ is null at the reference point, concave for gains and convex for losses, steeper for losses than for gains (see Figure 1).

The other important idea in CPT is the notion of probability distortion: people overweight very small probabilities and underweight average and large ones. This probability transformation is mathematically described by means of a weighting function, that is a strictly increasing function $\pi:[0 ; 1] \rightarrow[0 ; 1]$ satisfying the conditions $\pi(0)=0, \pi(1)=1$. A typical inverse S-shape weighting function graph is shown in Figure 2.

If in CPT two different weighting functions have the role to transform the probabilities attached to gains and losses, in our model we have a two-variables bi-weighting function. This has, in the first argument the probability of a gain with a utility greater or equal than a given level $L$ and in the second argument the probability of a symmetric loss, which utility is not smaller than $-L$. The

\footnotetext{
${ }^{1}$ which the authors called value function
} 


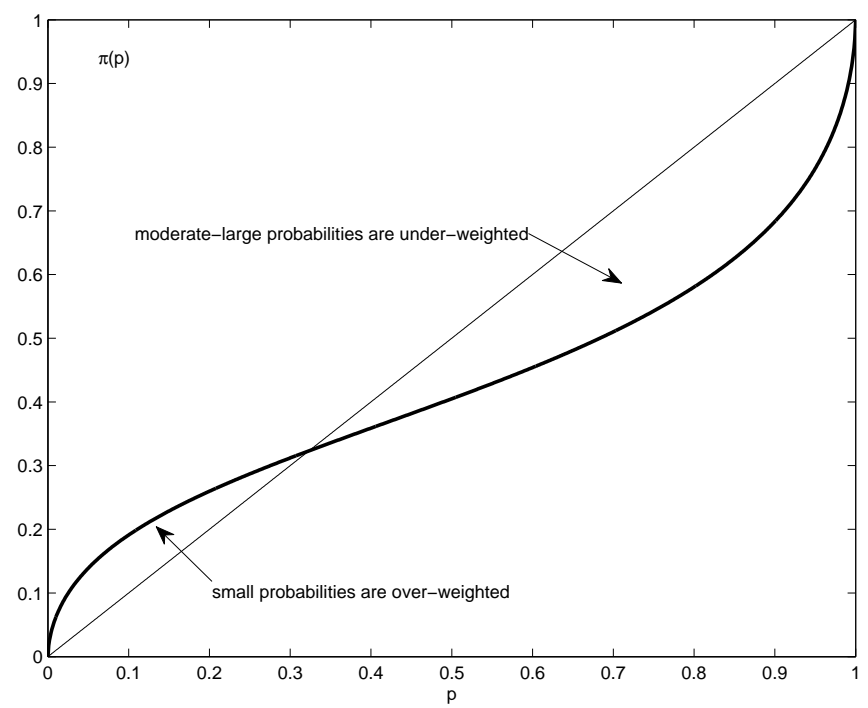

Figure 2: CPT weighting function

final result is a number within the closed interval $[-1 ; 1]$. Formally, let us set

$$
\mathcal{A}=\{(p, q) \in[0 ; 1] \times[0 ; 1] \text { such that } p+q \leq 1\},
$$

that is, in the $p-q$ plane, the triangle which vertexes are $O \equiv(0,0), P \equiv(1,0)$ and $Q \equiv(0,1)$.

Definition 1. We define bi-weighting function any function

$$
\omega(p, q): \mathcal{A} \rightarrow[-1 ; 1]
$$

satisfying the following coherence conditions:

- $\omega(p, q)$ is increasing in $p$ and decreasing in $q$ (bi-monotonicity)

- $\omega(1,0)=1, \omega(0,1)=-1$ and $\omega(0,0)=0$.

Let $\mathcal{P}=\left(x_{1}, p_{1} ; \ldots ; x_{n}, p_{n}\right)$ be a lottery assigning the outcome $x_{j} \in \mathfrak{R}$ with probability $p_{j}$, a utility function $u(\cdot): \Re \rightarrow \Re$, two weighting functions $\pi_{-}, \pi_{+}$ and a bi-weighting function $\omega$. Using an integral representation we can represent $\mathrm{CPT}$ and bCPT respectively as

$$
V_{C P T}(\mathcal{P})=\int_{0}^{+\infty} \pi_{+}\left(\sum_{i: u\left(x_{i}\right) \geq t} p_{i}\right) d t-\int_{0}^{+\infty} \pi_{-}\left(\sum_{i: u\left(x_{i}\right) \leq-t} p_{i}\right) d t
$$




$$
V_{b C P T}(\mathcal{P})=\int_{0}^{+\infty} \omega\left(\sum_{i: u\left(x_{i}\right) \geq t} p_{i}, \sum_{i: u\left(x_{i}\right) \leq-t} p_{i}\right) d t
$$

In our opinion, both these integrals genuinely generalize the original PT of Kahneman and Tversky (1979), preserving the main features of the theory. The only difference is that, in (1) we get a separate evaluation of gains and losses, whereas in (2) we get a conjoint evaluation. As we will soon see, the two formulas coincide in a non-mixed context, i.e. when the outcomes involved in the choice process are only gains or only loss. However, in the mixed case the two formulas can differ.

\section{The bi-weighting function}

In this section we propose some generalizations of well known weighting functions. They coincide with the original gain weighting function, $\pi_{+}$, if $q=0$, and with the opposite loss weighting function, $-\pi_{-}$, if $p=0$.

\subsection{The Kahneman-Tversky bi-weighting function}

The first and most famous weighting function was proposed in Tversky and Kahneman (1992):

$$
\pi(p)=\frac{p^{\gamma}}{\left[p^{\gamma}+(1-p)^{\gamma}\right]^{\frac{1}{\gamma}}}
$$

The parameter $\gamma$ can be chosen differently for gains and losses and the authors estimated $\gamma=0.61$ for gains and $\gamma=0.69$ for losses. For this weighting function we propose the following bipolar form

$$
\omega(p, q)=\frac{p^{\gamma}-q^{\delta}}{\left[p^{\gamma}+(1-p)^{\gamma}\right]^{\frac{1}{\gamma}}+\left[q^{\delta}+(1-q)^{\delta}\right]^{\frac{1}{\delta}}-1}
$$

As the original KT weighting function is non monotonic for $\gamma$ too much near to zero, - see Rieger and Wang (2006), Ingersoll (2008) - so it is the case of (3) when $\gamma$ and $\delta$ are near zero. Proposition 1 establishes the parameter limitations preserving the bi-monotonicity of (3). The proof is presented in appendix.

Proposition 1. The Kahneman, Tversky bi-weighting function with parameters $1 / 2<\gamma, \delta<1$, is increasing in $p$ and decreasing in $q$. 


\begin{tabular}{ccc}
\hline authors & $\alpha$ & $\gamma$ \\
\hline Tversky and Fox (1995) & 0.77 & 0.79 \\
Wu and Gonzalez (1996) & 0.84 & 0.68 \\
Gonzalez and Wu (1999) & 0.77 & 0.44 \\
Abdellaoui (2000) (gains) & 0.65 & 0.60 \\
Abdellaoui (2000) (losses) & 0.84 & 0.65 \\
Bleichrodt and Pinto (2000) & 0.816 & 0.550 \\
\hline
\end{tabular}

Table 1: recent estimations of parameters for the (4)

\subsection{The Latimore, Baker and Witte bi-weighting function}

Lattimore et al. (1992) and Goldstein and Einhorn (1987) introduced the following weighting function (with $\gamma, \alpha>0$ ) :

$$
\pi(p)=\frac{\alpha p^{\gamma}}{\alpha p^{\gamma}+(1-p)^{\gamma}}
$$

It is known as linear in log odd form, since Gonzalez and Wu (1999) proved this property. We propose the following bipolar form:

$$
\omega(p, q)=\frac{\alpha\left(p^{\gamma}-q^{\delta}\right)}{\alpha p^{\gamma}+(1-p)^{\gamma}+\alpha q^{\delta}+(1-q)^{\delta}-1}
$$

Proposition 2 (proof in appendix) establishes the parameter limitations allowing for the bi-monotonicity of (5). These limitations include many of previous parameter estimations given for the (4) (see table 1, from Bleichrodt and Pinto $(2000))$.

Proposition 2. The Latimore, Baker and Witte bi-weighting function with $\alpha>1 / 2$ and $0<\gamma, \delta \leq 1$, is increasing in $p$ and decreasing in $q$.

\subsection{The Prelec bi-weighting function}

One of the most famous alternative to the classical weighting function of Tversky and Kahneman (1992) is the compound-invariant form of Prelec (1998):

$$
\pi(p)=e^{-\beta(-L n p)^{\alpha}}
$$

where $\beta \approx 1$ is variable for gains and for losses and $0<\alpha<1$. The Prelec weighting function is undefined for $p=0$ but it is extended by continuity to the 
value of zero. We propose the following bi-weighting form:

$$
\omega(p, q)= \begin{cases}\frac{p^{\gamma}-q^{\delta}}{\left|p^{\gamma}-q^{\delta}\right|} e^{-\beta\left(-l n\left|p^{\gamma}-q^{\delta}\right|\right)^{\alpha}} & \forall(p, q) \in \mathcal{A} \mid p^{\gamma}-q^{\delta} \neq 0 \\ 0 & \forall(p, q) \in \mathcal{A} \mid p^{\gamma}-q^{\delta}=0\end{cases}
$$

The term $\frac{p^{\gamma}-q^{\delta}}{\left|p^{\gamma} q^{\delta}\right|}$ gives \pm 1 , respectively within the $O B A$ or $O B C$ "triangle" of figure 4. The (7) is extended by continuity when $p^{\gamma}-q^{\delta}=0$. Moreover the two parameters $\gamma$ and $\delta$ have the obvious motivation that we do not wish that $\omega(p, p)=0$ necessarily. Note that $\left|p^{\gamma}-q^{\delta}\right| \in[0,1]$ and then the logarithm is non positive. Proposition 3 establishes the parameters limitations allowing for the bi-monotonicity of (7). Without loss of generality, in the proof (see appendix) we choose $\beta=1$.

Proposition 3. The Prelec bi-weighting function with $\beta \cong 1, \gamma, \delta>0$ and $0<\alpha<1$ is increasing in $p$ and decreasing in $q$.

\subsection{The inverse S-shape of the bi-weighting function}

A typical feature of the weighting function described in Tversky and Kahneman (1992) is the inverse S-shape in the plane. Let us consider and plot the bipolarized form of the KT weighting function, preserving the original parameters estimation $\gamma=.61$ and $\delta=.69$

$$
\omega(p, q)=\frac{p^{0.61}-q^{0.69}}{\left[p^{0.61}+(1-p)^{0.61}\right]^{\frac{1}{0.61}}+\left[q^{0.69}+(1-q)^{0.69}\right]^{\frac{1}{0.69}}-1}
$$

The typical inverse S-Shape is generalized from the plane to the space (see Figure 3). Clearly we are interested to the part of this plot such that $p+q \leq 1$.

\subsection{Stochastic dominance and bCPT}

The bi-monotonicity of the bi-weighting function, ensures the bCPT model satisfies Stochastic Dominance Principle. This means that, if prospect $P$ stochastically dominates prospect $Q$, then $V_{b C P T}(P) \geq V_{b C P T}(Q)$. The following theorem establishes this result.

Theorem 1. Let us suppose that prospects are evaluated with the bipolar CPT. Then Stochastic Dominance Principle is satisfied.

Proof. Let us consider two lotteries $P=\left(x_{1}, p_{1} ; x_{2}, p_{2} ; \ldots ; x_{n}, p_{n}\right)$ and $Q=$ $\left(y_{1}, q_{1} ; y_{2}, q_{2} ; \ldots ; y_{m}, q_{m}\right)$ such that $P$ stochastically dominates $Q$. This means 


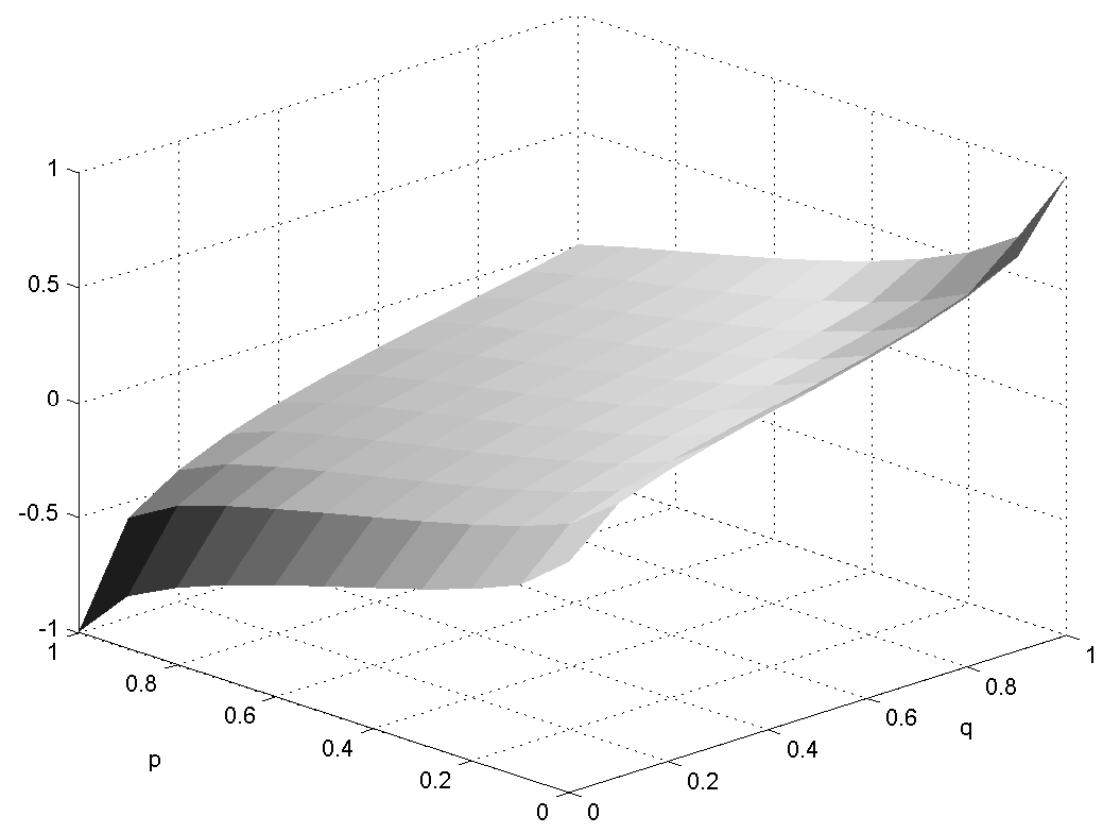

Figure 3: bi-CPT weighting function

that for all $t \in \mathfrak{R}$

$$
\sum_{i: x_{i} \geq t} p_{i} \geq \sum_{i: y_{i} \geq t} q_{i} \quad \text { or equivalently } \sum_{i: x_{i} \leq t} p_{i} \leq \sum_{i: y_{i} \leq t} q_{i}
$$

By the stochastic dominance of $P$ over $Q$, we have that for all $t \in \mathfrak{R}^{+}$

$$
\sum_{i: u\left(x_{i}\right) \geq t} p_{i} \geq \sum_{i: u\left(y_{i}\right) \geq t} q_{i} \text { and } \sum_{i: u\left(x_{i}\right) \leq-t} p_{i} \leq \sum_{i: u\left(y_{i}\right) \leq t} q_{i}
$$

From (10), considering the monotonicity of $\omega(\cdot, \cdot)$, we have that for all $t \in \mathfrak{R}^{+}$

$$
\omega\left(\sum_{i: u\left(x_{i}\right) \geq t} p_{i}, \sum_{i: u\left(x_{i}\right) \leq-t} p_{i}\right) \geq \omega\left(\sum_{i: u\left(y_{i}\right) \geq t} q_{i}, \sum_{i: u\left(y_{i}\right) \leq-t} q_{i}\right)
$$

and by monotonicity of the integral we conclude that $V_{b C P T}(P) \leq V_{b C P T}(Q)$.

On the other hand, in absence of the bi-monotonicity of the bi-weighting function we are able to build preferences violating the stochastic dominance. In fact, let us suppose the bi-weighting function $\omega(\cdot, \cdot)$ is not-[increasing in $p$ and 
decreasing in $q]$, i.e. that there exist $(p, q),(\widetilde{p}, \widetilde{q}) \in[0,1]^{2}$ such that

$$
\left\{\begin{array}{l}
p \geq \widetilde{p} \\
q \leq \widetilde{q} \\
(p-\widetilde{p})^{2}+(q-\widetilde{q})^{2}>0 \\
\omega(p, q)<\omega(\widetilde{p}, \widetilde{q})
\end{array}\right.
$$

Let us consider $x>0$ and $y<0$ such that $u(x)=-u(y)$ and the two lotteries $R=(x, p ; y, q)$ and $S=(x, \widetilde{p} ; y, \widetilde{q})$. Even if $R$ stochastically dominates $S$, it would results

$$
V_{b C P T}(R)=\omega(p, q) \cdot u(x)<\omega(\widetilde{p}, \widetilde{q}) \cdot u(x)=V_{b C P T}(S) .
$$

\section{The relationship between CPT and bCPT}

Given a bi-weighting function, $\omega(p, q): \mathcal{A} \rightarrow[-1 ; 1]$, it is straightforward to note that we can define two weighting functions by setting for all $p, q \in[0,1]$

$$
\begin{gathered}
\pi_{+}(p)=\omega(p, 0):[0,1] \rightarrow[0,1] \\
\pi_{-}(q)=-\omega(0, q):[0,1] \rightarrow[0,1]
\end{gathered}
$$

On the converse, given two weighting functions $\pi_{+}(p)$ and $\pi_{-}(q)$ we obtain a separable bi-weighting function by setting for all $(p, q) \in \mathcal{A}$

$$
\omega(p, q)=\pi_{+}(p)-\pi_{-}(q): \mathcal{A} \rightarrow[-1 ; 1]
$$

The next two propositions formalize the relationship between the two models.

Proposition 4. For non mixed prospects (containing only gains or losses) the bCPT model coincides with the CPT model.

Proof. Let us suppose that prospects are evaluated with bCPT and let $u(\cdot)$ : $\mathfrak{R} \rightarrow \mathfrak{R}$ be the utility function and $\omega(p, q): \mathcal{A} \rightarrow[-1,1]$ the bi-weighting function. Define the two weighting function $\pi_{+}(p)=\omega(p, 0)$ and $\pi_{-}(q)=-\omega(0, q)$ for all $p, q \in[0,1]$. Let $P=\left(x_{1}, p_{1} ; \ldots ; x_{n}, p_{n}\right)$ be a prospect assigning the non-negative outcome $x_{j} \in \mathfrak{R}^{+}$with probability $p_{j}$, we get:

$$
V_{b C P T}(P)=\int_{0}^{+\infty} \omega\left(\sum_{i: u\left(x_{i}\right) \geq t} p_{i}, \sum_{i: u\left(x_{i}\right) \leq-t} p_{i}\right) d t=
$$




$$
=\int_{0}^{+\infty} \omega\left(\sum_{i: u\left(x_{i}\right) \geq t} p_{i}, 0\right) d t=\int_{0}^{+\infty} \pi_{+}\left(\sum_{i: u\left(x_{i}\right) \geq t} p_{i}\right) d t=V_{C P T}(P)
$$

In the same manner, if $P=\left(x_{1}, p_{1} ; \ldots ; x_{n}, p_{n}\right)$ is a prospect assigning the nonpositive outcome $x_{j} \in \mathfrak{R}^{-}$with probability $p_{j}$, using $\omega(0, q)=-\pi_{-}(q)$ we get $V_{b C P T}(P)=V_{C P T}(P)$. Now let us suppose that prospects are evaluated with the CPT model and let us indicate with $u(\cdot): \mathfrak{R} \rightarrow \mathfrak{R}$ the utility function and with $\pi_{+}(p), \pi_{-}(q)$ the two weighting functions. By using the bi-weighting function $\omega(p, q)=\pi_{+}(p)-\pi_{-}(q)$ and replacing the steps in the above proof we get $V_{C P T}(P)=V_{b C P T}(P)$.

Proposition 4 states that CPT and bCPT are the same model for non-mixed prospects. This fact is, for us, of great importance, since CPT has been widely tested in situations involving only gains or only losses, as remembered for instance in $\mathrm{Wu}$ and Markle (2008): "In the last 50 years, a large body of empirical research has investigated how decision makers choose among risky gambles. Most of these findings can be accommodated by prospect theory... However, the majority of the existing empirical evidence has involved single-domain gambles.

Proposition 5. If the prospects are evaluated with the bCPT model with a separable bi-weighting function, then the representation coincides with that obtained with the CPT model. On the converse, if the prospects are evaluated with the CPT model, than the representation coincides with that obtained with the bCPT model with a separable bi-weighting function.

Proof. Let us suppose that prospects are evaluated with the bCPT model, with a separable bi-weighting function $\omega(p, q)=\pi_{+}(p)-\pi_{-}(q): \mathcal{A} \rightarrow[-1 ; 1]$. We get immediately:

$$
\begin{gathered}
V_{b C P T}(P)=\int_{0}^{+\infty} \omega\left(\sum_{i: u\left(x_{i}\right) \geq t} p_{i}, \sum_{i: u\left(x_{i}\right) \leq-t} p_{i}\right) d t= \\
=\int_{0}^{+\infty} \pi_{+}\left(\sum_{i: u\left(x_{i}\right) \geq t} p_{i}\right)-\pi_{-}\left(\sum_{i: u\left(x_{i}\right) \leq-t} p_{i}\right) d t=V_{C P T}(P)
\end{gathered}
$$

The converse is trivially obtained reversing the above steps.

Proposition 5 establishes that CPT can be considered a special case of bCPT, provided that we use a separable bi-weighting function. In other words there exists a (separable) bi-weighting function $\omega(p, q)=\pi_{+}(p)-\pi_{-}(q)$ such that $V_{b C P T}(P)=V_{C P T}(P)$ for all prospects $P$. This fact is relevant in order to 
provide a preference foundation for the model, since bCPT will need a less restrictive set of axioms with respect to CPT.

\subsection{BCPT and the Wu-Markle paradox}

Let us reconsider the Wu-Markle paradox described in the introduction. The paradox consists in the GLS violation, contrary to the prediction of CPT. Wu and Markle (2008) suggested to use the same model, CPT, with a different parametrization for mixed prospects and those involving only gains or losses: "Our study indicates that mixed gamble behavior is described well by an S-shaped utility function and an inverse S-shaped probability weighting function. However, gain-loss separability fails, and hence different parameter values are needed for mixed gambles than single-domain gambles..."

Despite these conclusions, we are able to explain their paradox using bCPT, without changing the parameters in the passage from non mixed prospects to mixed ones. If we use the bCPT with the bi-polarized KT weighting functions:

$$
\omega(p, q)=\frac{p^{0.61}-q^{0.69}}{\left[p^{0.61}+(1-p)^{0.61}\right]^{\frac{1}{0.61}}+\left[q^{0.69}+(1-q)^{0.69}\right]^{\frac{1}{0.69}}-1}
$$

and the classical KT power utility function ${ }^{2}$

$$
u(x)= \begin{cases}x^{.88} & \text { if } x \geq 0 \\ -2.25|-x|^{.88} & \text { if } x<0\end{cases}
$$

we obtain

$$
\begin{array}{ccc}
V_{b C P T}(\mathcal{H})=-443.24 & >V_{b C P T}(\mathcal{L})=-453.76 \\
V_{b C P T}\left(\mathcal{H}^{+}\right)=649.19 & <V_{b C P T}\left(\mathcal{L}^{+}\right)=652.26 \\
V_{b C P T}\left(\mathcal{H}^{-}\right)=-1,172.45 & <V_{b C P T}\left(\mathcal{L}^{-}\right)=-1,083.04
\end{array}
$$

These results agree with the preference relation $\gtrsim$. Wu and Markle (2008) is the most influential paper showing systematic violation of GLS. Similar results are, for example, in Birnbaum and Bahra (2007). In the appendix 2 we show in detail how bCPT seems to naturally capture the essence of the phenomenon.

\footnotetext{
${ }^{2}$ Both for $\omega(p, q)$ and $u(x)$ we use the the original parameters, estimated in Tversky and Kahneman (1992)
} 


\section{Extension of bCPT to uncertainty}

\subsection{Bi-capacity and the bipolar Choquet integral}

In order to extend bCPT to the field of uncertainty, we need to generalize the concept of capacity and Choquet integral with respect to a capacity. Let $\mathcal{S}$ be a non-empty set of states of the world and $\Sigma$ an algebra of subsets of $\mathcal{S}$ (the events). Let $\mathcal{B}$ denote the set of bounded real-valued $\Sigma$-measurable functions on $\mathcal{S}$ and $\mathcal{B}_{0}$ the set of simple (i.e. finite valued) functions in $\mathcal{B}$. A function $\nu: \Sigma \rightarrow[0,1]$ is a normalized capacity on $\Sigma$ if $\nu(\varnothing)=0, \nu(\mathcal{S})=1$ and $\nu(A) \leq \nu(B)$ whenever $A \subseteq B$. Choquet (1953) defined an integration operation with respect to $\nu$. Given a nonnegative valued function $f \in \mathcal{B}$ and a capacity $\nu: \Sigma \rightarrow[0,1]$, the Choquet integral of $f$ with respect to $\nu$ is

$$
\int_{S} f(s) d \nu=: \int_{0}^{\infty} \nu(\{s \in S: f(s) \geq t\}) d t
$$

Successively Schmeidler (1986) extended this definition to all of $\mathcal{B}$ :

$$
\int_{S} f(s) d \nu=: \int_{-\infty}^{0}[\nu(\{s \in S: f(s) \geq t\})-1] d t+\int_{0}^{\infty} \nu(\{s \in S: f(s) \geq t\}) d t
$$

Let us consider the set of all the couples of disjoint events

$$
\mathcal{Q}=\left\{(A, B) \in 2^{S} \times 2^{S}: A \cap B=\varnothing\right\}
$$

Definition 2. A function $\mu_{b}: \mathcal{Q} \rightarrow[-1,1]$ is a bi-capacity on $S$ if

- $\mu_{b}(\varnothing, \varnothing)=0, \mu_{b}(S, \varnothing)=1$ and $\mu_{b}(\varnothing, S)=-1$

- $\mu_{b}(A, B) \leq \mu_{b}(C, D)$ for all $(A, B),(C, D) \in \mathcal{Q}$ such that $A \subseteq C \wedge B \supseteq D$

Grabisch and Labreuche (2005a,b); Greco et al. (2002)

Definition 3. The bipolar Choquet integral of a simple function $f \in \mathcal{B}_{0}$ with respect to a bi-capacity $\mu_{b}$ is given by:

$$
\int_{S} f(s) d \mu_{b}=: \int_{0}^{\infty} \mu_{b}(\{s \in S: f(s)>t\},\{s \in S: f(s)<-t\}) d t
$$

Grabisch and Labreuche (2005a,b); Greco et al. (2002) 


\subsection{Two different approaches}

Since we are working with simple acts $f \in \mathcal{B}_{0}$, it follows that an uncertain act can be expressed as a vector $f=\left(x_{1}, s_{1} ; \cdots ; x_{n}, s_{n}\right)$, where $x_{i}$ will be obtained if the state $s_{i}$ will occur. Let $f_{+}$be the positive part of $f$, i.e. $f_{+}(s)=f(s)$ if

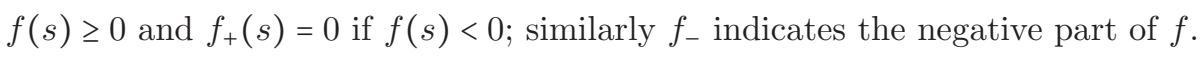
The dual capacity of a capacity $\nu: \Sigma \rightarrow[0,1]$ is defined as $\widehat{\nu}(A)=1-\nu\left(A^{c}\right)$ for all $A \in \Sigma$. Let be given an utility function $u(\cdot): \mathfrak{R} \rightarrow \mathfrak{R}$, two capacities (one for gains, one for losses) $\nu_{+}: \mathcal{S} \rightarrow[0,1]$ and $\nu_{-}: \mathcal{S} \rightarrow[0,1]$ and and a bi-capacity $\mu_{b}: \mathcal{Q} \rightarrow[-1,1]$. The evaluation of $f=\left(x_{1}, s_{1} ; \cdots ; x_{n}, s_{n}\right)$ in CPT and bCPT is

$$
\begin{gathered}
V_{C P T}(f)=\int_{\mathcal{S}} u\left[f_{+}(s)\right] d \nu_{+}+\int_{\mathcal{S}} u\left[f_{-}(s)\right] d \widehat{\nu}_{-}= \\
=\int_{0}^{\infty} \nu_{+}\left(\left\{s_{j}: u\left(x_{j}\right) \geq t\right\}\right) d t-\int_{-\infty}^{0} \nu_{-}\left(\left\{s_{i}: u\left(\left(x_{i}\right) \leq t\right\}\right) d t\right. \\
V_{b C P T}(\mathcal{P})=\int_{\mathcal{S}} u[f(s)] d \mu_{b}=\int_{0}^{+\infty} \mu_{b}\left(\left\{s_{i}: u\left(x_{i}\right)>t\right\},\left\{s_{i}: u\left(x_{i}\right)<-t\right\}\right) d t
\end{gathered}
$$

In CPT we sum the Choquet integral of $u\left(f_{+}\right)$with respect to $\nu_{+}$with the Choquet integral of $u\left(f_{-}\right)$with respect to $\widehat{\nu_{-}}$, by getting a separate evaluation of gains and losses. In bCPT we calculate the bipolar Choquet integral of $u(f)$ with respect to $\mu_{b}$ getting a conjointly evaluation of gains and losses.

\subsection{Link between CPT and bCPT}

As in a risk-context, the two situations where the two model coincide will occur for non mixed acts or by using a separable bi-capacity. If $\mu_{b}: \mathcal{Q} \rightarrow[-1,1]$ is a bi-capacity, then we can define two capacities $\nu_{+}$and $\nu_{-}$as follows: for all $E \in \Sigma$

$$
\begin{gathered}
\nu_{+}(E)=\mu_{b}(E, \varnothing) \\
\nu_{-}(E)=-\mu_{b}(\varnothing, E)
\end{gathered}
$$

If $f \in \mathcal{B}_{0}$ is such that $f(s) \geq 0$ for all $s \in S$, then

$$
\begin{gathered}
\int_{S} f(s) d \mu_{b}=\int_{0}^{\infty} \mu_{b}(\{s \in S: f(s)>t\}, \varnothing) d t= \\
=\int_{0}^{\infty} \nu_{+}(\{s \in S: f(s)>t\}) d t=\int_{S} f(s) d \nu_{+}
\end{gathered}
$$


If $f \in \mathcal{B}_{0}$ is such that $f(s) \leq 0$ for all $s \in S$, then

$$
\begin{aligned}
& \int_{S} f(s) d \mu_{b}=\int_{0}^{\infty} \mu_{b}(\varnothing,\{s \in S: f(s)<-t\}) d t= \\
& =-\int_{0}^{\infty} \nu_{-}(\{s \in S: f(s)<-t\}) d t=\int_{\mathcal{S}}(f(s)) d \widehat{\nu}_{-}
\end{aligned}
$$

We have established the following important relationship between CPT and bCPT:

Proposition 6. For non-mixed acts, the bCPT model coincides with the CPT model.

On the other hand, let us consider two capacities $\nu_{+}: \mathcal{S} \rightarrow[0,1]$ and $\nu_{-}: \mathcal{S} \rightarrow$ $[0,1]$. A separable bi-capacity is defined by setting for all $(A, B) \in \mathcal{Q}$

$$
\mu_{b}(A, B)=\nu_{+}(A)-\nu_{-}(B)
$$

Proposition 7. The bCPT model with a separable bi-weighting function coincides with the CPT model.

In fact, the bipolar Choquet integral with respect to a separable bi-capacity is the sum of two Choquet integrals. Let $f \in \mathcal{B}_{0}$ be a simple function and $\mu_{b}(A, B)=\nu_{+}(A)-\nu_{-}(B)$ a separable bi-weighting function, we get

$$
\begin{gathered}
\int_{S} f(s) d \mu_{b}=: \int_{0}^{\infty} \mu_{b}(\{s \in S: f(s)>t\},\{s \in S: f(s)<-t\}) d t= \\
=\int_{0}^{\infty}\left[\nu_{+}(\{s \in S: f(s)>t\})-\nu_{-}(\{s \in S: f(s)<-t\}) d t\right]= \\
=\int_{0}^{\infty} \nu_{+}(\{s \in S: f(s)>t\}) d t-\int_{0}^{\infty} \nu_{-}(\{s \in S: f(s)<-t\}) d t= \\
\int_{S} f_{+}(s) d \nu_{+}+\int_{S} f_{-}(s) d \widehat{\nu}_{-}
\end{gathered}
$$

In the remaining part of this paper we will face the problem of the preference foundation of bCPT. As we have just seen, the main concept to extend bCPT from the field of risk to that of uncertainty is the bipolar Choquet integral with respect to a bi-capacity. We will present a fairly simple characterization of the bipolar Choquet integral 


\section{The characterization theorem}

In this section, we first remark that the bipolar Choquet integral can be regarded as an extension of the bi-capacity. Next, we give the concept of absolutely comonotonic and co-signed acts, which are the special acts for which the functional is additive. Finally, we will state our main result, i.e. the characterization theorem.

Let us identify $(A, B) \in \mathcal{Q}$ with the bipolar-indicator function $(A, B)^{*} \in \mathcal{B}_{0}$

$$
(A, B)^{*}(s)=\left\{\begin{array}{cl}
1 & \text { if } s \in A \\
-1 & \text { if } s \in B \\
0 & \text { if } s \notin A \cup B
\end{array}\right.
$$

Since

$$
\int_{\mathcal{S}}(A, B)^{*} \mu_{b}=\int_{0}^{1} \mu_{b}(A, B) d t=\mu_{b}(A, B)
$$

then, the functional $\int_{\mathcal{S}} \mu_{b}$, i.e. the bipolar Choquet integral, can be considered as an extension of the bi-capacity $\mu_{b}$ from $\mathcal{Q}$ to $\mathcal{B}_{0}$.

Definition 4. $f, g: \mathcal{S} \rightarrow \mathfrak{R}$ are absolutely co-monotonic and cosigned (a.c.c.) if

- their absolute values are co-monotonic, i.e.

$$
(|f(s)|-|f(t)|) \cdot(|g(s)|-|g(t)|) \geq 0 \quad \forall s, t \in \mathcal{S}
$$

- they are co-signed, i.e.

$$
f(s) \cdot g(s) \geq 0 \quad \forall s \in \mathcal{S}
$$

Let us suppose that $\mu_{b}$ is a bi-capacity and let us indicate with $\mathcal{I}(f)=$ $\int_{\mathcal{S}} f(s) \mu_{b}$ the bipolar Choquet integral of $f$ with respect to $\mu_{b}$. The next proposition lists the properties of $\mathcal{I}$, and the following Theorem 2 characterizes $\mathcal{I}$. Given to the importance of this section, the proofs are presented in the main text.

Proposition 8. The functional $\mathcal{I}$ satisfies the following properties

- (P1) Monotonicity.

$$
f(s) \geq g(s) \quad \forall s \in \mathcal{S} \quad \Rightarrow \quad \mathcal{I}(f) \geq \mathcal{I}(g)
$$


- (P2) Positive homogeneity. For all $a>0$, and $f, a \cdot f \in \mathcal{B}_{0}$

$$
\mathcal{I}(a \cdot f)=a \cdot \mathcal{I}(f)
$$

- (P3) Bipolar-idem-potency. For all $\lambda>0$

$$
\mathcal{I}\left(\lambda(\mathcal{S}, \varnothing)^{*}\right)=\lambda \quad \text { and } \quad \mathcal{I}\left(\lambda(\varnothing, \mathcal{S})^{*}\right)=-\lambda
$$

- (P4) Additivity for acts a.c.c. If $f, g \in \mathcal{B}_{0}$ are a.c.c., then

$$
\mathcal{I}(f+g)=\mathcal{I}(f)+\mathcal{I}(g)
$$

Proof. Supposing $f(s) \geq g(s)$ for all $s \in \mathcal{S}$, then $\{s: f(s)>t\} \supseteq\{s: g(s)>t\}$ and $\{s \in S: f(s)<-t\} \subseteq\{s \in S: g(s)<-t\}$ such that (P1) follows from monotonicity of bicapacity and integral.

For all $a>0$ and for all $f \in B_{0}, a f \in B_{0}$, taking $t=a z$, by definition we get

$$
\begin{aligned}
& I(a f)=\int_{0}^{\infty} \mu_{b}\left(\left\{s \in S: f(s)>\frac{t}{a}\right\},\left\{s \in S: f(s)<-\frac{t}{a}\right\}\right) d t= \\
& \int_{0}^{\infty} \mu_{b}(\{s \in S: f(s)>z\},\{s \in S: f(s)<-z\}) a d z=a I(f) .
\end{aligned}
$$

which is $(\mathrm{P} 2)$.

For $\gamma>0$, by homogeneity, $I\left(\gamma(S, \varnothing)^{*}\right)=\gamma I(S, \varnothing)^{*}=\gamma \mu_{b}(S, \varnothing)=\gamma$.

If $\gamma<0$, then $I\left(\gamma(S, \varnothing)^{*}\right)=-\gamma I(\varnothing, S)^{*}=-\gamma \mu_{b}(\varnothing, S)=\gamma$. Note also that $I\left(0(S, \varnothing)^{*}\right)=I\left((\varnothing, \varnothing)^{*}\right)=\mu_{b}(\varnothing, \varnothing)=0$. Since $I\left(\lambda(\varnothing, S)^{*}\right)=-\lambda$ can be obtained analogously, thus (P3) is proved.

Let $f, g \in B_{0}$ be two acts a.c.c., then, generalizing remark 4 in Schmeidler (1986), there exist

- a partition of $S$ into $k$ pairwise disjoint subsets of $S,\left(E_{i}\right)_{i=1}^{k}$, such that for each $E_{i}$ there exist $E_{i}^{+}$and $E_{i}^{-}$with $E_{i}^{+} \cup E_{i}^{-}=E_{i}$ and $E_{i}^{+} \cap E_{i}^{-}=\varnothing$

- two $k$-list of numbers $0 \leq \alpha_{1} \leq \alpha_{2} \leq \cdots \leq \alpha_{k}$ and $0 \leq \beta_{1} \leq \beta_{2} \leq \cdots \leq \beta_{k}$

such that

$$
f=\sum_{i=1}^{k} \alpha_{i}\left(E_{i}^{+}, E_{i}^{-}\right)^{*} \quad, \quad g=\sum_{i=1}^{k} \beta_{i}\left(E_{i}^{+}, E_{i}^{-}\right)^{*}
$$

It follows that

$$
f+g=\sum_{i=1}^{k}\left(\alpha_{i}+\beta_{i}\right)\left(E_{i}^{+}, E_{i}^{-}\right)^{*}
$$


By the definition of bipolar Choquet integral,

$$
I(f+g)=I(f)+I(g)
$$

Theorem 2. Let $\mathcal{J}: \mathcal{B}_{0} \rightarrow \mathfrak{R}$ satisfy

- $\mathcal{J}\left((S, \varnothing)^{*}\right)=1$ and $\mathcal{J}\left((\varnothing, S)^{*}\right)=-1$;

- (P1) Monotonicity;

- (P4) Additivity for acts a.c.c.;

then, by assuming $\mu_{b}(A, B)=\mathcal{J}\left[(A, B)^{*}\right] \quad \forall(A, B) \in \mathcal{Q}$, we have

$$
\mathcal{J}(f)=\mathcal{I}(f)=\int_{S} f(s) d \mu_{b} \quad \forall f \in \mathcal{B}_{0}
$$

Proof. Let $f \in B_{0}$ be a simple function with image $f(S)=\left\{x_{1}, x_{2}, \ldots, x_{n}\right\}$. Let $(\cdot): N \rightarrow N$ be a permutation of indexes in $N=\{1,2, \ldots, n\}$ such that $\left|x_{(1)}\right| \leq\left|x_{(2)}\right| \leq \cdots \leq\left|x_{(n)}\right| . f$ can be written as sum of double-indicator functions, i.e.

$$
f=\sum_{i=1}^{n}\left(\left|x_{(i)}\right|-\left|x_{(i-1)}\right|\right)\left(A(f)_{(i)}, B(f)_{(i)}\right)^{*}
$$

where $A(f)_{(i)}=\left\{s \in S: f(s) \geq\left|x_{(i)}\right|\right\}, \quad B(f)_{(i)}=\left\{s \in S: f(s) \leq-\left|x_{(i)}\right|\right\}$ and $\left|x_{(0)}\right|=0$.

Observe that the simple functions $\left(A(f)_{(i)}, B(f)_{(i)}\right)^{*}$ for $i=1,2, \ldots, n$ are a.c.c., as well as the simple functions $\left(\left|x_{(i)}\right|-\left|x_{(i-1)}\right|\right)\left(A(f)_{(i)}, B(f)_{(i)}\right)^{*}$ for $i=1,2, \ldots, n$. On the basis of this observation, applying (P4), homogeneity and the definition of $\mu_{b}(A, B)$ we get the thesis as follows:

$$
\begin{gathered}
J(f)=J\left[\sum_{i=1}^{n}\left(\left|x_{(i)}\right|-\left|x_{(i-1)}\right|\right)\left(A(f)_{(i)}, B(f)_{(i)}\right)^{*}\right]= \\
=\sum_{i=1}^{n} J\left[\left(\left|x_{(i)}\right|-\left|x_{(i-1)}\right|\right)\left(A(f)_{(i)}, B(f)_{(i)}\right)^{*}\right]= \\
=\sum_{i=1}^{n}\left(\left|x_{(i)}\right|-\left|x_{(i-1)}\right|\right) J\left[\left(A(f)_{(i)}, B(f)_{(i)}\right)^{*}\right]= \\
=\sum_{i=1}^{n}\left(\left|x_{(i)}\right|-\left|x_{(i-1)}\right|\right) \mu_{b}\left(A(f)_{(i)}, B(f)_{(i)}\right)=\int_{S} f d \mu_{b}
\end{gathered}
$$


Remark 1. The properties (P2), i.e. the positive homogeneity, (P3) the bipolar idem-potency, are not among the hypothesis of Theorem 2 since they are implied by additivity for absolutely co-monotonic and cosigned acts (P4) and monotonicity (P1).

Remark 2. The fact that the functional, $\mathcal{I}$, is additive for a.c.c. functions, means that in the bCPT model the weakened version of independence axiom will be true for a.c.c. acts.

\section{Separating tastes from beliefs}

\subsection{Coherence conditions.}

The bipolar Choquet integral should represent preference under uncertainty. In this case it is reasonable to expect that there is some belief about plausibility of events $A \subseteq \mathcal{S}$ that should not depend on what is gained or lost in other events. In this context it is reasonable to imagine that the value given by a bi-capacity $\mu_{b}$ to $(A, B) \in Q$ is not decreasing with the plausibility of $A$ and non-increasing with the plausibility of $B$. If this is true, then one has to expect that should not be possible to have $\mu_{b}(A, C)>\mu_{b}(B, C)$ and $\mu_{b}(A, D)<\mu_{b}(B, D)$. In fact, this would mean that act $(A, C)^{*}$ would be preferred to act $(B, C)^{*}$, revealing a greater credibility of $A$ over $B$, and act $(A, D)^{*}$ would be preferred to act $(B, D)^{*}$, revealing a greater credibility of $B$ over $A$. Similar situations arise when $\mu_{b}(C, A)>\mu_{b}(C, B)$ and $\mu_{b}(D, A)<\mu_{b}(D, B)$, or $\mu_{b}(A, C)>\mu_{b}(B, C)$ and $\mu_{b}(D, A)>\mu_{b}(D, B)$. Taking into account such situations, we shall analyze in detail the following coherence conditions:

(A1) $(A, C)^{*}>(B, C)^{*} \Rightarrow(A, D)^{*}>(B, D)^{*}$, for all $(A, C),(B, C),(A, D),(B, D) \in Q$,

(A2) $(C, A)^{*}>(C, B)^{*} \Rightarrow(D, A)^{*}>(D, B)^{*}$, for all $(C, A),(C, B),(D, A),(D, B) \in Q$,

(A3) for any $A, B \subseteq S$ there exist one $C \subseteq S \backslash(A \cup B)$ such that $(A, C)^{*}>(B, C)^{*} \Leftrightarrow(C, A)^{*}<(C, B)^{*}$

(A4) $(A, C)^{*}>(B, C)^{*} \Leftrightarrow(C, A)^{*}<(C, B)^{*}$, for all $(A, C),(B, C),(C, A),(C, B) \in Q$,

(A5) $(A, C)^{*}>(B, C)^{*} \Leftrightarrow(D, A)^{*}<(D, B)^{*}$, for all $(A, C),(B, C),(D, A),(D, B) \in Q$. 
Theorem 3. The following proposition hold

1) If (A1) holds, then there exists a capacity $\nu_{1}$ on $S$ and a function

$$
\omega_{1}:\left\{(v, B): v=\nu_{1}(A),(A, B) \in Q\right\} \rightarrow[-1,1],
$$

such that $\mu_{b}(A, B)=\omega_{1}\left(\nu_{1}(A), B\right)$ for all $(A, B) \in Q$, with function $\omega_{1}$ increasing in the first argument and non increasing with respect to inclusion in the second argument;

2) If (A2) holds, then there exists a capacity $\nu_{2}$ on $S$ and a function

$$
\omega_{2}:\left\{(A, v): v=\nu_{2}(B),(A, B) \in Q\right\} \rightarrow[-1,1],
$$

such that $\mu_{b}(A, B)=\omega_{2}\left(A, \nu_{2}(B)\right)$ for all $(A, B) \in Q$, with function $\omega_{2}$ non decreasing with respect to inclusion in the first argument and decreasing in the second argument;

3) If (A1) and (A2) hold, then there exist two capacities $\nu_{1}$ and $\nu_{2}$ on $S$ and a function

$$
\omega_{3}:\left\{(u, v): u=\nu_{1}(A), v=\nu_{2}(B),(A, B) \in Q\right\} \rightarrow[-1,1]
$$

such that $\mu_{b}(A, B)=\omega_{3}\left(\nu_{1}(A), \nu_{2}(B)\right)$ for all $(A, B) \in Q$, with function $\omega_{3}$ increasing in the first argument and decreasing in the second argument;

4) If (A1), (A2) and (A3) hold, then there exists a capacity $\nu$ on $S$ and a function

$$
\omega:\{(u, v): u=\nu(A), v=\nu(B),(A, B) \in Q\} \rightarrow[-1,1],
$$

such that $\mu_{b}(A, B)=\omega(\nu(A), \nu(B))$ for all $(A, B) \in Q$, with function $\omega$ increasing in the first argument and decreasing in the second argument;

5) If (A1) and (A4) hold, then there exists a capacity $\nu$ on $S$ and a function

$$
\omega:\{(u, v): u=\nu(A), v=\nu(B),(A, B) \in Q\} \rightarrow[-1,1],
$$

such that $\mu_{b}(A, B)=\omega(\nu(A), \nu(B))$ for all $(A, B) \in Q$, with function $\omega$ increasing in the first argument and decreasing in the second argument; 
6) If (A2) and (A4) hold, then there exists a capacity $\nu$ on $S$ and a function

$$
\omega:\{(u, v): u=\nu(A), v=\nu(B),(A, B) \in Q\} \rightarrow[-1,1]
$$

such that $\mu_{b}(A, B)=\omega(\nu(A), \nu(B))$ for all $(A, B) \in Q$, with function $\omega$ increasing in the first argument and decreasing in the second argument;

7) If (A5) holds, then there exists a capacity $\nu$ on $S$ and a function

$$
\omega:\{(u, v): u=\nu(A), v=\nu(B),(A, B) \in Q\} \rightarrow[-1,1]
$$

such that $\mu_{b}(A, B)=\omega(\nu(A), \nu(B))$ for all $(A, B) \in Q$, with function $\omega$ increasing in the first argument and decreasing in the second argument.

The proof is presented in appendix 3 .

\section{Concluding Remarks}

In bCPT, gains and losses within a mixed prospect are evaluated conjointly and not separately, as in CPT. This permits to account for situations in which CPT fails, due to gain-loss separability, such as the "Wu-Markle paradox". In this paper we propose a natural generalization of $\mathrm{CPT}$, which, fundamentally: a) totally preserve CPT in non-mixed cases; b) allows for GLS violation in mixed case. The main concept to get an axiomatic foundation of bCPT, in decision under uncertainty, is the bipolar Choquet integral, about which, we have presented a fairly simple characterization. A full axiomatization of the model, in terms of preferences foundation, will be the aim for future researches.

\section{References}

Abdellaoui, M. (2000). Parameter-free elicitation of utility and probability weighting functions. Management Science, 46(11), 1497-1512.

Allais, M. (1953). Le comportement de l'homme rationnel devant le risque: Critique des postulats et axiomes de l'école Américaine. Econometrica: Journal of the Econometric Society, 21(4), 503-546.

Baltussen, G., Post, T., and van Vliet, P. (2006). Violations of cumulative 
prospect theory in mixed gambles with moderate probabilities. Management Science, $\mathbf{5 2}(8), 1288$.

Barberis, N., Huang, M., and Santos, T. (2001). Prospect Theory and Asset Prices. Quarterly Journal of Economics, 116(1), 1-53.

Benartzi, S. and Thaler, R. (1995). Myopic loss aversion and the equity premium puzzle. The Quarterly Journal of Economics, 110(1), 73-92.

Birnbaum, M. (2005). Three new tests of independence that differentiate models of risky decision making. Management Science, 51(9), 1346-1358.

Birnbaum, M. and Bahra, J. (2007). Gain-loss separability and coalescing in risky decision making. Management Science, 53(6), 1016-1028.

Blavatskyy, P. (2005). Back to the St. Petersburg paradox? Management Science, 51(4), 677-678.

Bleichrodt, H. and Pinto, J. (2000). A parameter-free elicitation of the probability weighting function in medical decision analysis. Management Science, 46(11), 1485-1496.

Camerer, C. (2000). Prospect theory in the wild: Evidence from the field. Advances in Behavioral Economics.

Choquet, G. (1953). Theory of capacities. Ann. Inst. Fourier, 5(131-295), 54.

Ellsberg, D. (1961). Risk, ambiguity, and the Savage axioms. The Quarterly Journal of Economics, 75(4), 643-669.

Gilboa, I. (1987). Expected utility with purely subjective non-additive probabilities. Journal of Mathematical Economics, 16(1), 65-88.

Goldstein, W. and Einhorn, H. (1987). Expression theory and the preference reversal phenomena. Psychological Review, 94(2), 236.

Gonzalez, R. and Wu, G. (1999). On the shape of the probability weighting function. Cognitive Psychology, 38(1), 129-166.

Grabisch, M. and Labreuche, C. (2005a). Bi-capacities-I: definition, M

"obius transform and interaction. Fuzzy sets and systems, 151(2), 211-236.

Grabisch, M. and Labreuche, C. (2005b). Bi-capacities-II: the Choquet integral. Fuzzy sets and systems, 151(2), 237-259. 
Greco, S., Matarazzo, B., and Slowinski, R. (2002). Bipolar Sugeno and Choquet integrals. In EUROFUSE Workshop on Informations Systems, pages 191-196.

Ingersoll, J. (2008). Non-monotonicity of the Tversky-Kahneman probabilityweighting function: a cautionary note. European Financial Management, 14(3), 385-390.

Jolls, C., Sunstein, C., and Thaler, R. (1998). A behavioral approach to law and economics. Stanford Law Review, 50(5), 1471-1550.

Kahneman, D. and Tversky, A. (1979). Prospect theory: An analysis of decision under risk. Econometrica: Journal of the Econometric Society, 47(2), 263291.

Lattimore, P., Baker, J., and Witte, A. (1992). The influence of probability on risky choice:: A parametric examination. Journal of Economic Behavior $\mathcal{E}$ Organization, 17(3), 377-400.

Levy, M. and Levy, H. (2002). Prospect Theory: Much ado about nothing? Management Science, 48(10), 1334-1349.

Luce, R. (1999). Binary Gambles of a Gain and a Loss: an Understudied Domain. Mathematical utility theory: utility functions, models, and applicaitons in the social sciences, 8, 181-202.

Luce, R. (2000). Utility of Gains and Losses:: Measurement-Theoretical, and Experimental Approaches.

McNeil, B., Pauker, S., Sox Jr, H., and Tversky, A. (1982). On the elicitation of preferences for alternative therapies. New England journal of medicine, 306(21), 1259-1262.

Prelec, D. (1998). The probability weighting function. Econometrica, 66(3), $497-527$.

Quattrone, G. and Tversky, A. (1988). Contrasting rational and psychological analyses of political choice. The American political science review, 82(3), 719-736.

Quiggin, J. (1982). A theory of anticipated utility. Journal of Economic Behavior \& Organization, 3(4), 323-343. 
Rieger, M. and Wang, M. (2006). Cumulative prospect theory and the St. Petersburg paradox. Economic Theory, 28(3), 665-679.

Schade, C., Schroeder, A., and Krause, K. (2010). Coordination after gains and losses: Is prospect theory's value function predictive for games? Journal of Mathematical Psychology.

Schmeidler, D. (1986). Integral representation without additivity. Proceedings of the American Mathematical Society, 97(2), 255-261.

Schmeidler, D. (1989). Subjective probability and expected utility without additivity. Econometrica: Journal of the Econometric Society, 57(3), 571-587.

Tversky, A. and Fox, C. (1995). Weighing risk and uncertainty. Psychological review, 102(2), 269-283.

Tversky, A. and Kahneman, D. (1992). Advances in prospect theory: Cumulative representation of uncertainty. Journal of Risk and uncertainty, 5(4), 297-323.

Von Neumann, J. and Morgenstern, O. (1944). Theories of games and economic behavior. Princeton University Press Princeton, NJ.

Wu, G. and Gonzalez, R. (1996). Curvature of the probability weighting function. Management Science, 42(12), 1676-1690.

Wu, G. and Markle, A. (2008). An empirical test of gain-loss separability in prospect theory. Management Science, 54(7), 1322-1335. 


\section{Appendix 1}

Proof of proposition 1 .

For $x \in[0,1]$ and $\delta \in[0,1]$ it results $f(x)=\left[x^{\delta}+(1-x)^{\delta}\right]^{\frac{1}{\delta}} \geq 1$ since this function is continuous in the closed interval $[0,1]$, with $f(0)=f(1)=1$, while $f^{\prime}(x)$ is positive in $] 0,1 / 2[$ and negative in $] 1 / 2,1[$. In fact:

$$
\begin{gathered}
f^{\prime}(x)=\left[x^{\delta}+(1-x)^{\delta}\right]^{\frac{1}{\delta}-1}\left[x^{\delta-1}-(1-x)^{\delta-1}\right] \geq 0 \\
\Leftrightarrow\left[x^{\delta-1}-(1-x)^{\delta-1}\right] \geq 0 \Leftrightarrow 1 \geq\left(\frac{x}{1-x}\right)^{1-\delta} \Leftrightarrow x \leq \frac{1}{2}
\end{gathered}
$$

It follows that in (3) the denominator is positive and the sign depends on $p^{\gamma}-q^{\delta}$. If we start from the zero curve $\omega(p, q)=0 \Leftrightarrow p^{\gamma}-q^{\delta}=0$, that is the $\widehat{O B}$ curve in figure 4 , it is clear that an increase in $p$ will bring us in the domain in which the function (3) is positive (OAB "triangle") while an increase in $q$ will bring us in the domain in which the function is negative (OBC "triangle") and then, in this case, the function (3) is increasing in $p$ and decreasing in $q$. Now it is sufficient to prove that $\omega(p, q)$ is increasing in $p$ and decreasing in $q$ within the two triangles, i.e. where $\omega(p, q)>0(<0)$ and $p, q>0$. If $\omega(p, q)>0$, and then if $p^{\gamma}-q^{\delta}>0$ and since the function $\ln (x)$ is strictly increasing, it is sufficient to prove that $\ln [\omega(p, q)]$ is increasing in $p$ and decreasing in $q$. By differentiating w. r. t. the first variable:

$$
\begin{gathered}
\frac{\partial \ln [\omega(p, q)]}{\partial p}=\frac{\gamma p^{\gamma-1}}{p^{\gamma}-q^{\delta}}-\left[\left(\frac{1}{p}\right)^{1-\gamma}-\left(\frac{1}{1-p}\right)^{1-\gamma}\right] . \\
\frac{\left[p^{\gamma}+(1-p)^{\gamma}\right]^{\frac{1}{\gamma}-1}}{\left[p^{\gamma}+(1-p)^{\gamma}\right]^{\frac{1}{\gamma}}+\left[q^{\delta}+(1-q)^{\delta}\right]^{\frac{1}{\delta}}-1}
\end{gathered}
$$

If $1 / 2 \leq p<1 \rightarrow\left[\left(\frac{1}{p}\right)^{1-\gamma}-\left(\frac{1}{1-p}\right)^{1-\gamma}\right] \leq 0$ and (14) is positive. Suppose $0<p<1 / 2$, then the first summand in (14) is positive and the second is negative. We have the following decreasing sequence:

$$
\frac{\partial \ln [\omega(p, q)]}{\partial p}=\frac{\gamma p^{\gamma-1}}{p^{\gamma}-q^{\delta}}-\left[\left(\frac{1}{p}\right)^{1-\gamma}-\left(\frac{1}{1-p}\right)^{1-\gamma}\right] \text {. }
$$




$$
\begin{gathered}
\frac{\left[p^{\gamma}+(1-p)^{\gamma}\right]^{\frac{1}{\gamma}-1}}{\left[p^{\gamma}+(1-p)^{\gamma}\right]^{\frac{1}{\gamma}}+\left[q^{\delta}+(1-q)^{\delta}\right]^{\frac{1}{\delta}}-1} \geq^{3} \\
\geq \frac{\gamma p^{\gamma-1}}{p^{\gamma}}-\left[\left(\frac{1}{p}\right)^{1-\gamma}-\left(\frac{1}{1-p}\right)^{1-\gamma}\right] \cdot \frac{\left[p^{\gamma}+(1-p)^{\gamma}\right]^{\frac{1}{\gamma}-1}}{\left[p^{\gamma}+(1-p)^{\gamma}\right]^{\frac{1}{\gamma}}+\left[q^{\delta}+(1-q)^{\delta}\right]^{\frac{1}{\delta}}-1} \geq \\
\geq \\
=\frac{\gamma p^{\gamma-1}}{p^{\gamma}}-\left[\left(\frac{1}{p}\right)^{1-\gamma}-\left(\frac{1}{1-p}\right)^{1-\gamma}\right] \cdot \frac{\left[p^{\gamma}+(1-p)^{\gamma}\right]^{\frac{1}{\gamma}-1}}{\left[p^{\gamma}+(1-p)^{\gamma}\right]^{\frac{1}{\gamma}}}= \\
\geq \frac{\gamma\left(\frac{1}{p}\right)^{1-\gamma}}{p^{\gamma}}-\frac{\left(\frac{1}{p}\right)^{1-\gamma}}{p^{\gamma}+(1-p)^{\gamma}}=\left(\frac{1}{p}\right)^{1-\gamma} \cdot\left[\frac{\gamma}{p^{\gamma}}-\frac{1}{p^{\gamma}+(1-p)^{\gamma}}\right]
\end{gathered}
$$

Now, in order to prove that the (14) is non negative, it is sufficient to show that the quantity in the last square bracket is non negative, i.e.

$$
\begin{gathered}
\frac{\gamma}{p^{\gamma}}-\frac{1}{p^{\gamma}+(1-p)^{\gamma}}=\frac{\gamma\left[p^{\gamma}+(1-p)^{\gamma}\right]-p^{\gamma}}{p^{\gamma}\left[p^{\gamma}+(1-p)^{\gamma}\right]} \geq 0 \Leftrightarrow \gamma\left[p^{\gamma}+(1-p)^{\gamma}\right]-p^{\gamma} \geq 0 \\
\Leftrightarrow \gamma(1-p)^{\gamma} \geq(\gamma) p^{\gamma} \Leftrightarrow\left(\frac{1-p}{p}\right)^{\gamma} \geq \frac{1-\gamma}{\gamma} \Leftrightarrow \frac{1-p}{p} \geq\left(\frac{1-\gamma}{\gamma}\right)^{\frac{1}{\gamma}}
\end{gathered}
$$

Remembering that we are under the limitation $0<p<1 / 2$ the first term is

$$
\begin{aligned}
& { }^{3} \text { since } \\
& \frac{\gamma p^{\gamma-1}}{p^{\gamma}-q^{\delta}}>\frac{\gamma p^{\gamma-1}}{p^{\gamma}} \\
& { }^{4} \text { since from } \\
& {\left[q^{\delta}+(1-q)^{\delta}\right]^{\frac{1}{\delta}}-1 \geq 0 \rightarrow \frac{\left[p^{\gamma}+(1-p)^{\gamma}\right]^{\frac{1}{\gamma}-1}}{\left[p^{\gamma}+(1-p)^{\gamma}\right]^{\frac{1}{\gamma}}+\left[q^{\delta}+(1-q)^{\delta}\right]^{\frac{1}{\delta}}-1} \leq \frac{\left[p^{\gamma}+(1-p)^{\gamma}\right]^{\frac{1}{\gamma}-1}}{\left[p^{\gamma}+(1-p)^{\gamma}\right]^{\frac{1}{\gamma}}} \rightarrow} \\
& -\left[\left(\frac{1}{p}\right)^{1-\gamma}-\left(\frac{1}{1-p}\right)^{1-\gamma}\right] \frac{\left[p^{\gamma}+(1-p)^{\gamma}\right]^{\frac{1}{\gamma}-1}}{\left[p^{\gamma}+(1-p)^{\gamma}\right]^{\frac{1}{\gamma}}+\left[q^{\delta}+(1-q)^{\delta}\right]^{\frac{1}{\delta}}-1} \geq \\
& -\left[\left(\frac{1}{p}\right)^{1-\gamma}-\left(\frac{1}{1-p}\right)^{1-\gamma}\right] \frac{\left[p^{\gamma}+(1-p)^{\gamma}\right]^{\frac{1}{\gamma}-1}}{\left[p^{\gamma}+(1-p)^{\gamma}\right]^{\frac{1}{\gamma}}} \\
& -\left(\frac{1}{p}\right)^{1-\gamma} \leq-\left[\left(\frac{1}{p}\right)^{1-\gamma}-\left(\frac{1}{1-p}\right)^{1-\gamma}\right] \leq 0
\end{aligned}
$$


greater than 1 and the last inequality is true if

$$
\left(\frac{1-\gamma}{\gamma}\right)^{\frac{1}{\gamma}} \leq 1 \Leftrightarrow \gamma \geq \frac{1}{2}
$$

and this is ensured by the hypothesis of proposition 1 .

Thus we have proved that if $\omega(p, q)>0$ then the function $\omega(p, q)$ is increasing in $p$. An analogous proof gives that, if $\omega(p, q)<0$, then the function is decreasing in $q$, i.e. the function $-\omega(p, q)$ is increasing in $q$. For this it is sufficient to exchange $p$ with $q$ and $\gamma$ with $\delta$ and to repeat the previous passages. Now, in the case $\omega(p, q)>0$ we turn out our attention to the first derivative of $\ln [\omega(p, q)]$ with respect to $q$

$$
\begin{gathered}
\frac{\partial \ln [\omega(p, q)]}{\partial q}=\frac{-\delta q^{\delta-1}}{p^{\gamma}-q^{\delta}}-\left[\left(\frac{1}{q}\right)^{1-\delta}-\left(\frac{1}{1-q}\right)^{1-\delta}\right] . \\
\frac{\left[q^{\delta}+(1-q)^{\delta}\right]^{\frac{1}{\delta}-1}}{\left[p^{\gamma}+(1-p)^{\gamma}\right]^{\frac{1}{\gamma}}+\left[q^{\delta}+(1-q)^{\delta}\right]^{\frac{1}{\delta}}-1}
\end{gathered}
$$

If $\left[\left(\frac{1}{q}\right)^{1-\delta}-\left(\frac{1}{1-q}\right)^{1-\delta}\right] \geq 0 \Leftrightarrow q \leq 1 / 2$ then the (15) is negative. Supposing $q>1 / 2$, the first summand in (15) is negative and the second is positive. Note that if $\gamma \geq \delta$, the curve which equation is $p^{\gamma}-q^{\delta}=0$ coincides with the graph of the function $q=p^{\frac{\gamma}{\delta}}$ that is convex, like in figure 4, and within the domain

$$
A^{+}=\left\{(p, q) \in[0 ; 1] \times[0 ; 1] \text { such that } p+q \geq 1 \text { and } p^{\gamma}-q^{\delta}\right\}
$$

it is impossible that $q>1 / 2$ and so we have finished the proof. On the other hand, if $\gamma<\delta$ the graph of the function $q=p^{\frac{\gamma}{\delta}}$ is concave and within the domain $A^{+}$there are points such that $q>1 / 2$. For these reasons, from here we will suppose $q>1 / 2$ and $\gamma<\delta$ and we will refer to figure 5 .

From a sequence of increases it results:

$$
\frac{\partial \ln [\omega(p, q)]}{\partial q} \leq{ }^{6} \leq \frac{-\delta q^{\delta-1}}{p^{\gamma}-q^{\delta}}-\left[\left(\frac{1}{q}\right)^{1-\delta}-\left(\frac{1}{1-q}\right)^{1-\delta}\right]=
$$

\footnotetext{
${ }^{6}$ since from$$
1 / 2<\gamma, \delta \leq 1 \rightarrow\left[p^{\gamma}+(1-p)^{\gamma}\right]^{\frac{1}{\gamma}}-1 \geq 0 \text { and }\left[q^{\delta}+(1-q)^{\delta}\right]^{\frac{1}{\delta}} \geq 1 \rightarrow
$$$$
\frac{\left[q^{\delta}+(1-q)^{\delta}\right]^{\frac{1}{\delta}-1}}{\left[p^{\gamma}+(1-p)^{\gamma}\right]^{\frac{1}{\gamma}}+\left[q^{\delta}+(1-q)^{\delta}\right]^{\frac{1}{\delta}}-1} \leq \frac{\left[q^{\delta}+(1-q)^{\delta}\right]^{\frac{1}{\delta}-1}}{\left[q^{\delta}+(1-q)^{\delta}\right]^{\frac{1}{\delta}}}=\frac{1}{q^{\delta}+(1-q)^{\delta}} \leq 1
$$ 


$$
=q^{\delta-1}\left[\frac{-\delta}{p^{\gamma}-q^{\delta}}+\left(\frac{q}{1-q}\right)^{1-\delta}-1\right]
$$

Then it is sufficient to prove that

$$
\frac{-\delta}{p^{\gamma}-q^{\delta}}+\left(\frac{q}{1-q}\right)^{1-\delta}-1 \leq 0 \Leftrightarrow\left(\frac{q}{1-q}\right)^{1-\delta} \leq 1+\frac{-\delta}{p^{\gamma}-q^{\delta}}
$$

and this will follow from:

$$
\frac{q}{1-q} \leq 1+\frac{-\delta}{p^{\gamma}-q^{\delta}}
$$

since

$$
q>\frac{1}{2} \Rightarrow \frac{q}{1-q}>1 \Rightarrow\left(\frac{q}{1-q}\right)^{1-\delta} \Rightarrow \frac{q}{1-q}
$$

For our scope we must prove that

$$
\frac{q}{1-q} \leq 1+\frac{-\delta}{p^{\gamma}-q^{\delta}}
$$

Under the restrictions we are working with, it is possible to elicit some limitations of the variables $p, q, \gamma$ and $\delta$. We have supposed $p^{\gamma}-q^{\delta}>0, q>1 / 2$ and $\delta>\gamma$, that in figure 5 delimit the area ABC. Since the curvature of $p^{\gamma}-q^{\delta}=0$ is more accentuate when larger is the difference between $\gamma$ and $\delta$, a limit is, for us, the curve $p^{0.5}-q^{1}=0$, i.e. $q=\sqrt{p}$, which delimits the area ADE containing the area ABC. This consideration allows us to elicit some sure limitations for $p$ and $q$ : the "highest" point is the intersection between $q=\sqrt{p}$ and $p+q=1$, that is $D(0.38 ; 0.62)$; the most "left-placed" point is the intersection between $q=\sqrt{p}$ and $q=0.5$, that is $E(0.25 ; 0.5)$; we elicit $0.25<p<0.5$ and $0.5<q<0.62$. Consider the function $p^{\gamma}-q^{\delta}$, by differentiating, we can prove that it is increasing in $p$ and $\delta$ and decreasing in $q$ and $\gamma$, and then, using the elicited parameter limitations we have

$$
p^{\gamma}-q^{\delta} \leq\left(\frac{1}{2}\right)^{0.5}-\left(\frac{1}{2}\right)^{1}
$$

which in turn implies

$$
1+\frac{-\delta}{p^{\gamma}-q^{\delta}} \geq 1+\frac{\delta}{\left(\frac{1}{2}\right)^{0.5}-\left(\frac{1}{2}\right)^{1}}
$$

Finally, the quantity $q /(1-q)$ is increasing in $q$ and then by using the sup 
limitation of $q$ it follows that

$$
\frac{q}{1-q} \leq \frac{0.62}{1-0.62}
$$

Using (17) and (18) the (16) is true if it is true the:

$$
\frac{0.62}{1-0.62} \leq 1+\frac{\delta}{\left(\frac{1}{2}\right)^{0.5}-\left(\frac{1}{2}\right)^{1}}
$$

which gives $\delta>0.131$ that is within our limitations. Similarly, by exchanging $p$ with $q$ and $\gamma$ with $\delta$ it follows that $\omega(p, q)$ is increasing in $p$ when $\omega(p, q)<0$.

Q.E.D.

Proof of proposition 2.

For $x \in[0,1], \alpha>1 / 2$ and $\gamma \in] 0,1]$ it results $f(x)=\alpha x^{\gamma}+(1-x)^{\gamma} \geq \min \{1, \alpha\}>$ $1 / 2$. Since this function is continuous in the closed interval $[0,1]$, with $f(0)=1$, $f(1)=\alpha$ and the second derivative is non-positive from zero to one:

$$
f^{\prime \prime}(x)=\gamma(\gamma-1) \alpha x^{\delta-2}+\gamma(\gamma-1)(1-x)^{\delta-2} \leq 0
$$

It follows that, in (5), the denominator is positive under the limitation $\alpha>1 / 2$. Within its domain the first derivative of the (5) with respect to $p$ is :

$$
\frac{\partial \omega(p, q)}{\partial p}=\alpha \gamma \frac{(1-p)^{\gamma-1}\left(p^{\gamma-1}-q^{\delta}\right)+p^{\gamma-1}\left[2 \alpha q^{\delta}+(1-q)^{\delta}-1\right]}{\left[\alpha p^{\gamma}+(1-p)^{\gamma}+\alpha q^{\delta}+(1-q)^{\delta}-1\right]^{2}}
$$

Having chosen $\gamma \leq 1$ the term $p^{\gamma-1} \geq 1$ for all $\left.\left.p \in\right] 0,1\right]$ and since $q^{\delta} \leq 1$ then $p^{\gamma-1}-q^{\delta} \geq 0$. On the other hand $\left(2 \alpha q^{\delta}+1-q\right)^{\delta}-1 \geq 0$ since for $x \in[0,1]$, $\alpha>1 / 2$ and $0<\delta \leq 1$ the function $f(x)=2 \alpha x^{\delta}+(1-x)^{\delta} \geq \min \{1,2 \alpha\} \geq 1$ since it is continuous in the closed interval $[0,1]$, with $f(0)=1, f(1)=2 \alpha$ and the second derivative is non-positive from zero to one:

$$
f^{\prime \prime}(x)=\gamma(\gamma-1) 2 \alpha x^{\delta-2}+\gamma(\gamma-1)(1-x)^{\delta-2} \leq 0
$$

Then (19) is non-negative and the (5) is increasing in $p$.

The first derivative with respect to $q$ is

$$
\frac{\partial \omega(p, q)}{\partial q}=\alpha \delta \frac{(1-q)^{\delta-1}\left(p^{\gamma}-q^{\delta-1}\right)-q^{\delta-1}\left[2 \alpha p^{\gamma}+(1-p)^{\gamma}-1\right]}{\left[\alpha p^{\gamma}+(1-p)^{\gamma}+\alpha q^{\delta}+(1-q)^{\delta}-1\right]^{2}}
$$


By the same argumentations, it is easy to see that it is non-positive and then the (5) is decreasing in $q$.

Q.E.D.

\section{Proof of proposition 3.}

If we start from the zero curve $\omega(p, q)=0 \Leftrightarrow p^{\gamma}-q^{\delta}=0$ that is the $\widehat{O B}$ curve in figure 4 , it is clear that an increasing in $p$ will bring them in the domain in which the function is positive $(O A B$ "triangle") while an increasing in $q$ will bring them in the domain in which the function is negative $(O B C$ "triangle") and, in this case, the function (7) is increasing in $p$ and decreasing in $q$. Now it is sufficient to prove that $\omega(p, q)$ is increasing in $p$ and decreasing in $q$ within the two triangle, i.e. where $\omega(p, q)>0$ or $\omega(p, q)<0$ and $p, q>0$. If $w(p, q)>0$ and then if $p^{\gamma}-q^{\delta}>0$ the $(7)$ becomes: $\omega(p, q)=e^{-\left[-\operatorname{Ln}\left(p^{\gamma}-q^{\delta}\right)\right]^{\alpha}}$ and by differentiating w. r. t. the two variables:

$$
\begin{aligned}
& \frac{\partial \omega(p, q)}{\partial p}=e^{-\left[-\operatorname{Ln}\left(p^{\gamma}-q^{\delta}\right)\right]^{\alpha}} \alpha\left[-\operatorname{Ln}\left(p^{\gamma}-q^{\delta}\right)\right]^{\alpha-1} \frac{\gamma p^{\gamma-1}}{p^{\gamma}-q^{\delta}}>0 \\
& \frac{\partial \omega(p, q)}{\partial p}=e^{-\left[-\operatorname{Ln}\left(p^{\gamma}-q^{\delta}\right)\right]^{\alpha}} \alpha\left[-\operatorname{Ln}\left(p^{\gamma}-q^{\delta}\right)\right]^{\alpha-1} \frac{-\delta q^{\delta-1}}{p^{\gamma}-q^{\delta}}<0
\end{aligned}
$$

This proves the property within the triangle $O B A$, where $\omega(p, q)>0$. Similarly if $p^{\gamma}-q^{\delta}<0$ the $(7)$ becomes: $\omega(p, q)=-e^{-\left[-L n\left(-p^{\gamma}+q^{\delta}\right)\right]^{\alpha}}$ and by differentiating w. r. t. the two variables:

$$
\begin{aligned}
& \frac{\partial \omega(p, q)}{\partial p}=-e^{-\left[-\operatorname{Ln}\left(-p^{\gamma}+q^{\delta}\right)\right]^{\alpha}} \alpha\left[-\operatorname{Ln}\left(p^{\gamma}-q^{\delta}\right)\right]^{\alpha-1} \frac{-\gamma p^{\gamma-1}}{-p^{\gamma}+q^{\delta}}>0 \\
& \frac{\partial \omega(p, q)}{\partial p}=-e^{-\left[-\operatorname{Ln}\left(-p^{\gamma}+q^{\delta}\right)\right]^{\alpha}} \alpha\left[-\operatorname{Ln}\left(p^{\gamma}-q^{\delta}\right)\right]^{\alpha-1} \frac{\delta q^{\delta-1}}{-p^{\gamma}+q^{\delta}}<0
\end{aligned}
$$

We conclude that the Prelec bi-weighting function is increasing in its first argument and decreasing in the second, for all the parameter values.

Q.E.D. 


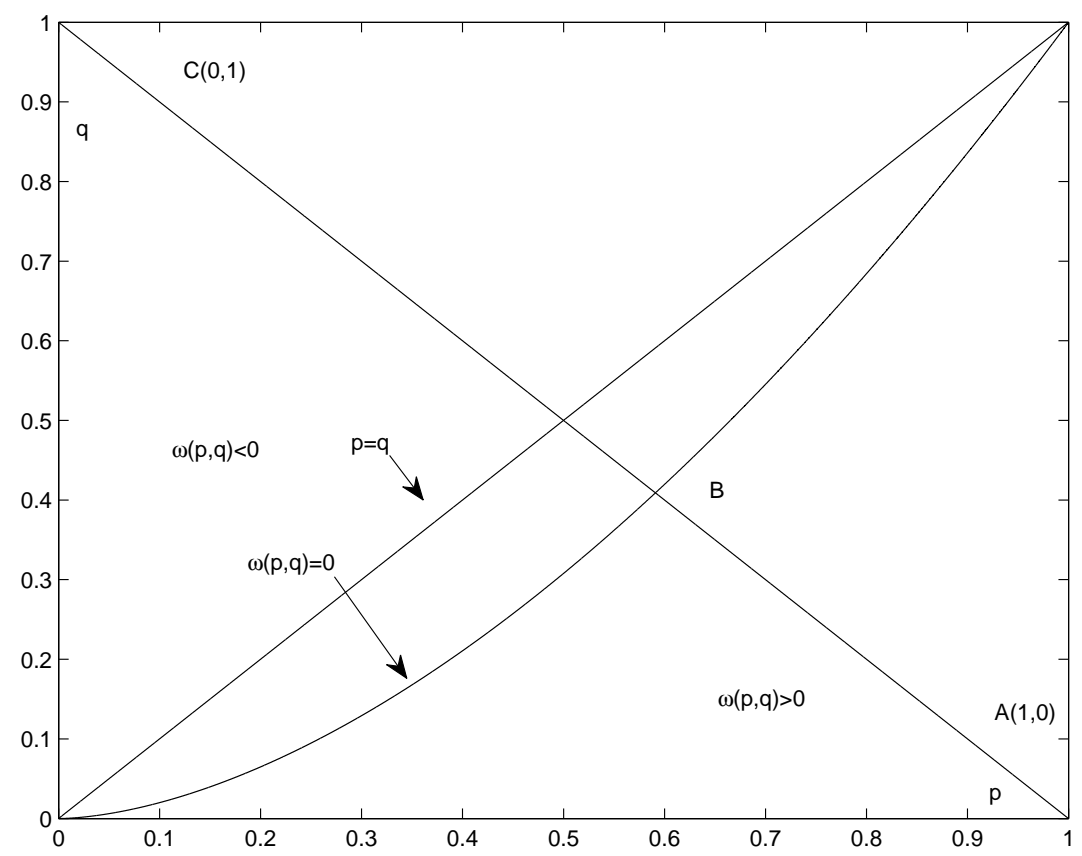

Figure 4: the KT bi-weighting function domain; in the case $\gamma>\delta$, the curve $q=p^{\gamma / \delta}$ is convex.

\section{Appendix 2}

\subsection{Recent literature denouncing GLS}

As discussed in the paper, this study aims to generalize CPT, allowing gains and losses within a mixed prospect to be evaluated conjointly, rather than separately. In the following we shall focus our attention on two recent papers: $\mathrm{Wu}$ and Markle (2008) and Birnbaum and Bahra (2007). Both of them report systematic violations of GLS. CPT and all the model it generalizes, such as EUT, cannot account for such a pattern of choice. We show how bCPT is able to capture, at least partially, these errata predictions.

\subsection{Wu and Markle (2008)}

In table 2 we reproduce Table 1 of page 1326 in Wu and Markle (2008), with the preferences elicited from the reported percentages found by the authors. In many cases (tests $6,7,10-18$ ) the respondents preferred (in percentage) $H$ to $L$ while, splitting the prospects into their respective positive and negative 
part, the preferences were reversed. To test our model we have used the bCPT functional

$$
V_{b C P T}(P)=\int_{0}^{\infty} \omega\left(\sum_{i: u\left(x_{i}\right) \geq t} p_{i}, \sum_{i: u\left(x_{i}\right) \leq-t} p_{i}\right) d t
$$

based on the KT bi-weighting function

$$
\omega(p, q)=\frac{p^{\gamma}-q^{\delta}}{\left[p^{\gamma}+(1-p)^{\gamma}\right]^{\frac{1}{\gamma}}+\left[q^{\delta}+(1-q)^{\delta}\right]^{\frac{1}{\delta}}-1}
$$

with parameters $\gamma=0.9$ and $\delta=0.89$ and the classical KT power utility function

$$
u(x)= \begin{cases}x_{+}^{\alpha} & \text { if } x \geq 0 \\ -\lambda(-x)_{-}^{\alpha} & \text { if } x<0\end{cases}
$$

with parameters $\lambda=1.77, \alpha_{+}=0.68$, and $\alpha_{-}=0.79$.

As can be seen in table 2, our data are in the same directions of the preferences in all the pure positive choices except that in tests 13, 23 and 25, in all the pure negative choices except in tests $9,12-15,17$ and 19 and in all the mixed choices except in tests 3,5 and 20. But, what we think is very interesting, is that bCPT is able explain the reversed preferences, totally in tests $6,7,10,11$, 16 and 18 and partially in test $12,14,15$, and 17 . The model seems able to naturally capture, totally or partially, the GLH.

\subsection{Birnbaum-Bahra}

Birnbaum and Bahra (2007) reported systematic violations of two behavioral properties implied by CPT. One, is the just discussed GLS and the other, is the property known as coalescing: "coalescing is the assumption that if there are two probability-consequences branches in a gamble leading to the same consequence, they can be combined by adding their probabilities." For example, the three-branch gamble $A=(\$ 100,25 \% ; \$ 100,25 \% ; \$ 0,50 \%)$ should be equivalent to the two-branch gamble $A^{\prime}=(\$ 100,50 \% ; \$ 0,50 \%)$. Our model is not able to accommodate for violation of coalescing, but we want some questions. Birnbaum and Bahra tested violation of coalescing presenting to the participants the gambles in terms of a container holding exactly 100 marbles of different colors. So, according to coalescing, $B^{\prime}=(25$ red $\$ 100 ; 75$ white $\$ 0)$ should be considered equivalent to $B=(25$ red $\$ 100 ; 25$ white $\$ 0 ; 50$ white $\$ 0)$. We are not sure that to present the gambles in this form, is the same that to present the 
Table 2: application of bCPT to the data of Wu and Markle (2008)

\begin{tabular}{|c|c|c|c|c|c|c|c|c|c|c|c|c|c|c|c|c|c|}
\hline \multirow[t]{2}{*}{ Test } & \multicolumn{4}{|c|}{$\mathrm{H}$ gamble } & \multicolumn{4}{|c|}{ L gamble } & \multicolumn{3}{|c|}{ choice $\%$} & \multirow{2}{*}{\multicolumn{3}{|c|}{ preferences }} & \multicolumn{3}{|c|}{$\mathrm{bCPT}$} \\
\hline & $\mathrm{g}$ & $\mathrm{p}$ & l & $1-p$ & $\mathrm{~g}^{\prime}$ & $\mathrm{p}^{\prime}$ & $\mathrm{l}^{\prime}$ & $1-\mathrm{p}^{\prime}$ & $\mathrm{H}$ & $\mathrm{H}+$ & $\mathrm{H}-$ & & & & & & \\
\hline 1 & 150 & 0,3 & -25 & 0,7 & 75 & 0,8 & -60 & 0,2 & 22 & 10 & 17 & $\mathrm{G}+$ & G- & G & $\mathrm{G}+$ & G- & G \\
\hline 2 & 1800 & 0,05 & -200 & 0,95 & 600 & 0,3 & -250 & 0,7 & 21 & 17 & 15 & $\mathrm{G}+$ & G- & G & $\mathrm{G}+$ & G- & G \\
\hline 3 & 1000 & 0,25 & -500 & 0,75 & 600 & 0,5 & -700 & 0,5 & 28 & 12 & 20 & $\mathrm{G}+$ & G- & G & $\mathrm{G}+$ & G- & 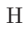 \\
\hline 4 & 200 & 0,3 & -25 & 0,7 & 75 & 0,8 & -100 & 0,2 & 33 & 18 & 22 & $\mathrm{G}+$ & G- & G & $\mathrm{G}+$ & G- & , \\
\hline 5 & 1200 & 0,25 & -500 & 0,75 & 600 & 0,5 & -800 & 0,5 & 43 & 21 & 25 & $\mathrm{G}+$ & G- & G & $\mathrm{G}+$ & G- & $\mathrm{H}$ \\
\hline 6 & 750 & 0,4 & -1000 & 0,6 & 500 & 0,6 & -1500 & 0,4 & 51 & 26 & 25 & $\mathrm{G}+$ & G- & HG & $\mathrm{G}+$ & G- & $\mathrm{H}$ \\
\hline 7 & 4200 & 0,5 & -3000 & 0,5 & 3000 & 0,75 & -6000 & 0,25 & 52 & 15 & 37 & $\mathrm{G}+$ & G- & HG & $\mathrm{G}+$ & G- & $\mathrm{H}$ \\
\hline 8 & 4500 & 0,5 & -1500 & 0,5 & 3000 & 0,75 & -3000 & 0,25 & 48 & 17 & 47 & $\mathrm{G}+$ & G- & GH & $\mathrm{G}+$ & G- & $\mathrm{H}$ \\
\hline 9 & 4500 & 0,5 & -3000 & 0,5 & 3000 & 0,75 & -6000 & 0,25 & 58 & 17 & 55 & $\mathrm{G}+$ & $\mathrm{H}-$ & $\mathrm{H}$ & $\mathrm{G}+$ & G- & $\mathrm{H}$ \\
\hline 10 & 1000 & 0,3 & -200 & 0,7 & 400 & 0,7 & -500 & 0,3 & 51 & 48 & 28 & $\mathrm{G}+$ & G- & HG & $\mathrm{G}+$ & G- & $\mathrm{H}$ \\
\hline 11 & 4800 & 0,5 & -1500 & 0,5 & 3000 & 0,75 & -3000 & 0,25 & 54 & 33 & 44 & $\mathrm{G}+$ & G- & $\mathrm{H}$ & $\mathrm{G}+$ & G- & $\mathrm{H}$ \\
\hline 12 & 3000 & 0,01 & -490 & 0,99 & 2000 & 0,02 & -500 & 0,98 & 59 & 42 & 36 & $\mathrm{G}+$ & G- & $\mathrm{H}$ & $\mathrm{G}+$ & H- & $\mathrm{H}$ \\
\hline 13 & 2200 & 0,4 & -600 & 0,6 & 850 & 0,75 & -1700 & 0,25 & 52 & 38 & 42 & $\mathrm{G}+$ & G- & HG & $\mathrm{H}+$ & H- & $\mathrm{H}$ \\
\hline 14 & 2000 & 0,2 & -1000 & 0,8 & 1700 & 0,25 & -1100 & 0,75 & 58 & 34 & 48 & $\mathrm{G}+$ & G- & $\mathrm{H}$ & $\mathrm{G}+$ & H- & $\mathrm{H}$ \\
\hline 15 & 1500 & 0,25 & -500 & 0,75 & 600 & 0,5 & -900 & 0,5 & 51 & 51 & 33 & GH+ & G- & HG & $\mathrm{H}+$ & H- & $\mathrm{H}$ \\
\hline 16 & 5000 & 0,5 & -3000 & 0,5 & 3000 & 0,75 & -6000 & 0,25 & 65 & 43 & 43 & $\mathrm{G}+$ & G- & $\mathrm{H}$ & $\mathrm{G}+$ & G- & $\mathrm{H}$ \\
\hline 17 & 1500 & 0,4 & -1000 & 0,6 & 600 & 0,8 & -3500 & 0,2 & 59 & 48 & 41 & $\mathrm{G}+$ & G- & $\mathrm{H}$ & $\mathrm{G}+$ & H- & $\mathrm{H}$ \\
\hline 18 & 2025 & 0,5 & -875 & 0,5 & 1800 & 0,6 & -1000 & 0,4 & 72 & 52 & 42 & $\mathrm{G}+$ & G- & $\mathrm{H}$ & $\mathrm{G}+$ & G- & $\mathrm{H}$ \\
\hline 19 & 600 & 0,25 & -100 & 0,75 & 125 & 0,75 & -500 & 0,25 & 58 & 55 & 44 & $\mathrm{H}+$ & G- & $\mathrm{H}$ & $\mathrm{H}+$ & H- & $\mathrm{H}$ \\
\hline 20 & 5000 & 0,1 & -900 & 0,9 & 1400 & 0,3 & -1700 & 0,7 & 40 & 47 & 53 & $\mathrm{G}+$ & HG- & G & $\mathrm{G}+$ & G- & $\mathrm{H}$ \\
\hline 21 & 700 & 0,25 & -100 & 0,75 & 125 & 0,75 & -600 & 0,25 & 71 & 59 & 48 & $\mathrm{H}+$ & H- & $\mathrm{H}$ & $\mathrm{H}+$ & G- & $\mathrm{H}$ \\
\hline 22 & 700 & 0,5 & -150 & 0,5 & 350 & 0,75 & -400 & 0,25 & 63 & 58 & 48 & $\mathrm{H}+$ & GH- & $\mathrm{H}$ & $\mathrm{H}+$ & H- & $\mathrm{H}$ \\
\hline 23 & 1200 & 0,3 & -200 & 0,7 & 400 & 0,7 & -800 & 0,3 & 70 & 59 & 50 & $\mathrm{H}+$ & H- & $\mathrm{H}$ & $\mathrm{G}+$ & H- & $\mathrm{H}$ \\
\hline 24 & 5000 & 0,5 & -2500 & 0,5 & 2500 & 0,75 & -6000 & 0,25 & 79 & 54 & 54 & $\mathrm{H}+$ & H- & $\mathrm{H}$ & $\mathrm{H}+$ & H- & $\mathrm{H}$ \\
\hline 25 & 800 & 0,4 & -1000 & 0,6 & 500 & 0,6 & -1600 & 0,4 & 58 & 64 & 51 & $\mathrm{H}+$ & H- & $\mathrm{H}$ & $\mathrm{G}+$ & H- & $\mathrm{H}$ \\
\hline 26 & 5000 & 0,5 & -3000 & 0,5 & 2500 & 0,75 & -6500 & 0,25 & 71 & 61 & 59 & $\mathrm{H}+$ & H- & $\mathrm{H}$ & $\mathrm{H}+$ & H- & $\mathrm{H}$ \\
\hline 27 & 700 & 0,25 & -100 & 0,75 & 100 & 0,75 & -800 & 0,25 & 73 & 58 & 64 & $\mathrm{H}+$ & H- & $\mathrm{H}$ & $\mathrm{H}+$ & H- & $\mathrm{H}$ \\
\hline 28 & 1500 & 0,3 & -200 & 0,7 & 400 & 0,7 & -1000 & 0,3 & 75 & 59 & 63 & $\mathrm{H}+$ & H- & $\mathrm{H}$ & $\mathrm{H}+$ & G- & $\mathrm{H}$ \\
\hline 29 & 1600 & 0,25 & -500 & 0,75 & 600 & 0,5 & -1100 & 0,5 & 73 & 60 & 69 & $\mathrm{H}+$ & $\mathrm{H}-$ & $\mathrm{H}$ & $\mathrm{H}+$ & H- & $\mathrm{H}$ \\
\hline 30 & 2000 & 0,4 & -800 & 0,6 & 600 & 0,8 & -3500 & 0,2 & 65 & 66 & 63 & $\mathrm{H}+$ & H- & $\mathrm{H}$ & $\mathrm{H}+$ & H- & $\mathrm{H}$ \\
\hline 31 & 2000 & 0,25 & -400 & 0,75 & 600 & 0,5 & -1100 & 0,5 & 80 & 63 & 69 & $\mathrm{H}+$ & H- & $\mathrm{H}$ & $\mathrm{H}+$ & H- & $\mathrm{H}$ \\
\hline 32 & 1500 & 0,4 & -700 & 0,6 & 300 & 0,8 & -3500 & 0,2 & 78 & 64 & 68 & $\mathrm{H}+$ & H- & $\mathrm{H}$ & $\mathrm{H}+$ & H- & $\mathrm{H}$ \\
\hline 33 & 900 & 0,4 & -1000 & 0,6 & 500 & 0,6 & -1800 & 0,4 & 70 & 74 & 61 & $\mathrm{H}+$ & H- & $\mathrm{H}$ & $\mathrm{H}+$ & H- & $\mathrm{H}$ \\
\hline 34 & 1000 & 0,4 & -1000 & 0,6 & 500 & 0,6 & -2000 & 0,4 & 78 & 71 & 70 & $\mathrm{H}+$ & $\mathrm{H}-$ & $\mathrm{H}$ & $\mathrm{H}+$ & H- & $\mathrm{H}$ \\
\hline
\end{tabular}


gambles with the numerical probabilities. In fact, a person facing $B$ could ask himself what is the reason that the first 25 white marbles were not summed to the second 50 white marbles. It is admissible that she could think if they differ in some way, e.g. in size. In any case, she will have an additional information, or doubt to process and this could generate errors. As focused from $\mathrm{Wu}$ and Markle (2008), the examples of Birnbaum and Bahra (2007) to underline the GLS violation, are less simple than theirs, but our model is able to accommodate for these violations too. The only we need is to modify the parameter $\gamma$ from the value of 0.9 , used to accommodate the majority of data in $\mathrm{Wu}$ and Markle (2008), to the value of 0.74. Next, we report the part of the table 5 at page 1022 in Birnbaum and Bahra (2007) that, in the words of the same authors, form a test for the GLS. Each gamble "is described in terms of a container holding exactly 100 marbles of different colors, from which one marble would be drawn at random, and the color of that marble would determine the prize.". In the brackets are shown the percentages of each choose.

$$
\begin{aligned}
& \mathcal{F}=\left(\begin{array}{c}
25 \text { black } \\
\text { to win } \$ 100 \\
25 \text { white } \\
\text { to win } \$ 0 \\
50 \text { pink } \\
\text { to lose } \$ 50
\end{array}\right)>\left(\begin{array}{c}
50 \text { blue } \\
\text { to win } \$ 50 \\
\\
25 \text { white } \\
\text { to lose } \$ 0 \\
25 \text { red } \\
\text { to lose } \$ 100
\end{array}\right)=\mathcal{G} \\
& \text { [76\%] [24\%] } \\
& \mathcal{F}^{+}=\left(\begin{array}{c}
25 \text { black } \\
\text { to win } \$ 100 \\
25 \text { white } \\
\text { to win } \$ 0 \\
50 \text { white } \\
\text { to win } \$ 0
\end{array}\right)<\left(\begin{array}{c}
25 \text { blue } \\
\text { to win } \$ 50 \\
\\
25 \text { blue } \\
\text { to win } \$ 50 \\
50 \text { white } \\
\text { to win } \$ 0
\end{array}\right)=\mathcal{G}^{+}
\end{aligned}
$$

[29\%] [71\%] 


$$
\mathcal{F}^{-}=\left(\begin{array}{c}
50 \text { white } \\
\text { to lose } \$ 0 \\
25 \text { pink } \\
\text { to lose } \$ 50 \\
25 \text { pink } \\
\text { to lose } \$ 50 \\
{[35 \%]}
\end{array}\right)<\left(\begin{array}{c}
50 \text { white } \\
\text { to lose } \$ 0 \\
25 \text { white } \\
\text { to lose } \$ 0 \\
25 \text { red } \\
\text { to lose } \$ 100 \\
{[65 \%]}
\end{array}\right)=\mathcal{G}^{-}
$$

As can be seen, $\mathcal{F}$ is preferred to $\mathcal{G}$, but when the two prospects are split in their respective positive and negative parts (according to coalescing) a relevant majority prefers $\mathcal{G}^{+}$to $\mathcal{F}^{+}$and $\mathcal{G}^{-}$to $\mathcal{F}^{-}$. In order to evaluate these prospects, we substitute the respective probabilities to the colors, as the authors did, by dividing for 100 any number of color. Using the bipolar CPT with the bipolarized KT weighting functions with parameters $\gamma=0.74, \delta=0.89$

$$
\omega(p, q)=\frac{p^{0.74}-q^{0.89}}{\left[p^{0.74}+(1-p)^{0.74}\right]^{\frac{1}{0.74}}+\left[q^{0.89}+(1-q)^{0.89}\right]^{\frac{1}{0.89}}-1}
$$

and the classical KT power utility function with parameters $\lambda=1.77, \alpha=0.68$ and $\beta=0.79$

$$
u(x)= \begin{cases}x^{0.68} & \text { if } x \geq 0 \\ -1.77(-x)^{0.79} & \text { if } x<0\end{cases}
$$

we obtain

$$
\begin{gathered}
V_{b C P T}(\mathcal{F})=-11.07 \geq V_{b C P T}(\mathcal{G})=-11.11 \\
V_{b C P T}\left(\mathcal{F}^{+}\right)=6.67 \leq V_{b C P T}\left(\mathcal{G}^{+}\right)=6.71 \\
V_{b C P T}\left(\mathcal{F}^{-}\right)=-19.28 \leq V_{b C P T}\left(\mathcal{G}^{-}\right)=-18.25
\end{gathered}
$$

These results agree with the preference relation $\gtrsim$.

\section{Appendix 3}

Proof of Theorem 3.

1) Let us define $\nu_{1}(A)=\mu_{b}(A, \varnothing)$. For all $(A, C),(B, C),(A, D),(B, D) \in Q$, it 


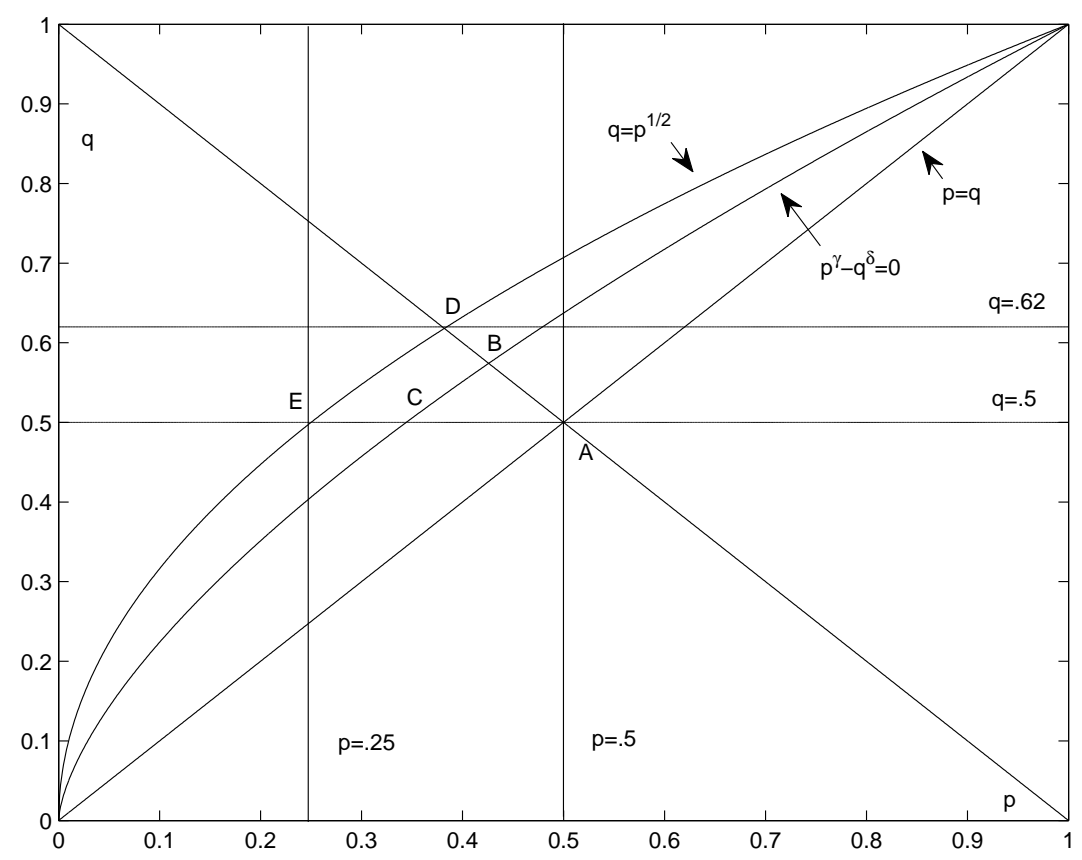

Figure 5: if $\gamma<\delta$ the curve $\widehat{O B}: q=p^{\gamma / \delta}$ is concave and its most accentuate curvature is that of $\widehat{O D}: q=\sqrt{p}$. The point $A(.5, .5)$ is the intersection between the lines $p=q$ and $p+q=1$; the point $B$ is the intersection between $p^{\gamma}-q^{\delta}=0$ and $p+q=1$; the point $C$ is the intersection between $p^{\gamma}-q^{\delta}=0$ and $q=.5$; the point $D(.38, .62)$ is the intersection between $q=\sqrt{p}$ and $p+q=1$; the point $E(.25, .5)$ is the intersection between $q=\sqrt{p}$ and $q=.5$.

is not possible to have $\mu_{b}(A, C)=\mu_{b}(B, C)$ and $\mu_{b}(A, D)>\mu_{b}(B, D)$, because, for $(\mathrm{A} 1), \mu_{b}(A, D)>\mu_{b}(B, D)$ would imply $\mu_{b}(A, C)>\mu_{b}(B, C)$, too. Thus,

$$
\mu_{b}(A, C)=\mu_{b}(B, C) \Rightarrow \mu_{b}(A, D)=\mu_{b}(B, D),
$$

for all $(A, C),(B, C),(A, D),(B, D) \in Q$. Consequently,

$$
\mu_{b}(A, \varnothing)=\mu_{b}(B, \varnothing) \Rightarrow \mu_{b}(A, C)=\mu_{b}(B, C)
$$

from which we get

$$
\nu_{1}(A)=\nu_{1}(B) \Rightarrow \mu_{b}(A, C)=\mu_{b}(B, C),
$$


for all $(A, C),(B, C) \in Q$. We can therefore define function $\omega_{1}$ as follows: $\omega_{1}\left(\nu_{1}(A), B\right)=\mu_{b}(A, B)$, for all $(A, B) \in Q$.

For (A1) we have

$$
\mu_{b}(A, \varnothing)>\mu_{b}(B, \varnothing) \Rightarrow \mu_{b}(A, C)>\mu_{b}(B, C)
$$

for all $(A, C),(B, C) \in Q$, i.e.

$$
\nu_{1}(A)>\nu_{1}(B) \Rightarrow \mu_{b}(A, C)>\mu_{b}(B, C),
$$

and consequently

$$
\nu_{1}(A)>\nu_{1}(B) \Rightarrow \omega_{1}\left(\nu_{1}(A), C\right)>\omega_{1}\left(\nu_{1}(B), C\right),
$$

which means that $\omega_{1}$ is increasing in the first argument. Monotonicity of bipolar capacity gives the monotonicity of function $\omega_{1}$ with respect to the second argument.

2) It can be proved analogously to 1$)$, by defining $\nu_{2}(A)=-\mu_{b}(\varnothing, A)$.

3) By 1) and 2).

4) Condition (A3) ensures that capacities $\nu_{1}$ and $\nu_{2}$ agree in the sense that for all $A, B \subseteq S$

$$
\nu_{1}(A)>\nu_{1}(B) \Leftrightarrow \nu_{2}(A)>\nu_{2}(B) .
$$

Indeed, applying the definition of $\nu_{1},(\mathrm{~A} 1),(\mathrm{A} 3),(\mathrm{A} 2)$ and the definition of $\nu_{2}$, we get

$$
\begin{gathered}
\nu_{1}(A)>\nu_{1}(B) \Rightarrow \mu_{b}(A, \varnothing)>\mu_{b}(B, \varnothing) \Rightarrow \\
\mu_{b}(A, C)>\mu_{b}(B, C) \Leftrightarrow \mu_{b}(C, A)<\mu_{b}(C, B) \\
\Rightarrow \mu_{b}(\varnothing, A)<\mu_{b}(\varnothing, B) \Rightarrow \nu_{2}(A)>\nu_{2}(B),
\end{gathered}
$$

i.e.

$$
\nu_{1}(A)>\nu_{1}(B) \Rightarrow \nu_{2}(A)>\nu_{2}(B) . \quad(i i)
$$


Analogously, applying the definition of $\nu_{2},(\mathrm{~A} 2),(\mathrm{A} 3),(\mathrm{A} 1)$ and the definition of $\nu_{1}$, we get

$$
\begin{gathered}
\nu_{2}(A)>\nu_{2}(B) \Rightarrow \mu_{b}(\varnothing, B)>\mu_{b}(\varnothing, A) \Rightarrow \\
\mu_{b}(C, A)>\mu_{b}(C, B) \Leftrightarrow \mu_{b}(A, C)<\mu_{b}(B, C) \\
\Rightarrow \mu_{b}(A, \varnothing)<\mu_{b}(B, \varnothing) \Rightarrow \nu_{1}(A)>\nu_{1}(B),
\end{gathered}
$$

i.e.

$$
\nu_{2}(A)>\nu_{2}(B) \Rightarrow \nu_{1}(A)>\nu_{1}(B) . \quad(i i i)
$$

By (i) and (ii) we get (iii). (iii) implies also that for all $A, B \subseteq S$

$$
\nu_{1}(A)=\nu_{1}(B) \Leftrightarrow \nu_{2}(A)=\nu_{2}(B) . \quad(i v)
$$

By (i) and (iv), there exists an increasing function $g:\{v \in[0,1]: \exists A \subseteq$ $S$ for which $\left.\nu_{2}(A)=v\right\} \rightarrow[0,1]$ such that $\nu_{2}(A)=g\left(\nu_{1}(A)\right)$. Thus we can define a function $\omega:\left\{(u, v): u=\nu_{1}(A), v=\nu_{1}(B),(A, B) \in Q\right\} \rightarrow[-1,1]$ defined as follows: for all $A, B) \in Q$

$$
\omega\left(\nu_{1}(A), \nu_{1}(B)\right)=\omega_{3}\left(\nu_{1}(A), g\left(\nu_{1}(B)\right)\right)
$$

where $\omega_{3}$ is the function defined in point 3 ). For the monotonicity of function $\omega_{3}$ and $g, \omega$ is increasing in the first argument and decreasing in the second argument.

5) Observe that (A3) is a particular case of (A4), which, given $A, B \subseteq S$ holds for any $C \subseteq S$ such that $(A, C),(B, C),(C, A),(C, B) \in Q$ (observe that if $(A, C),(B, C) \in Q$, then also $(C, A),(C, B) \in Q)$. Using (A4), (A1) and again (A4) we get (A2) as follows: for all $(C, A),(C, B),(A, D),(B, D) \in Q$

$$
\begin{aligned}
& \mu_{b}(C, A)>\mu_{b}(C, B) \Leftrightarrow \mu_{b}(A, C)<\mu_{b}(B, C) \Rightarrow \\
& \Rightarrow \mu_{b}(A, D)<\mu_{b}(B, D) \Leftrightarrow \mu_{b}(D, A)>\mu_{b}(D, B)
\end{aligned}
$$


With (A1), (A2) and (A3) we can apply 4) and obtain the result we looked for.

6) Analogously to 5), using (A4), (A2) and again (A4) we get (A1) as follows: for all $(C, A),(C, B),(A, D),(B, D) \in Q$

$$
\begin{gathered}
\mu_{b}(A, C)>\mu_{b}(B, C) \Leftrightarrow \mu_{b}(C, A)<\mu_{b}(C, B) \Rightarrow \\
\mu_{b}(D, A)<\mu_{b}(D, B) \Leftrightarrow \mu_{b}(A, D)>\mu_{b}(B, D)
\end{gathered}
$$

With (A1), (A2) and (A3) we can apply 4) and obtain the result we looked for.

7) (A4) is a specific case of (A5), which, given $A, B \subseteq S$ holds for any $C, D \subseteq S$ such that $(A, C),(B, C),(D, A),(D, B) \in Q$. Using (A4), (A1) can obtained as follows:

$$
\mu_{b}(A, C)>\mu_{b}(B, C) \Leftrightarrow \mu_{b}(D, A)<\mu_{b}(D, B) \Leftrightarrow \mu_{b}(A, D)>\mu_{b}(B, D) .
$$

With (A1) and (A4) we can apply 5) and we get the thesis.

Q.E.D. 